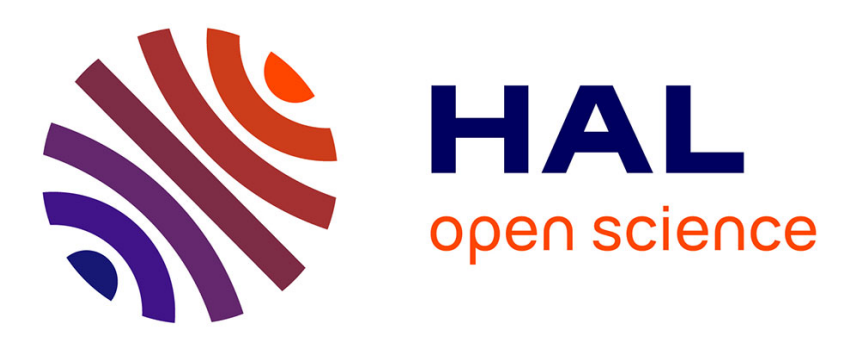

\title{
Mathematical modeling of electromagnetic wave propagation in heterogeneous lossy coaxial cables with variable cross section
}

Sébastien Imperiale, Patrick Joly

\section{- To cite this version:}

Sébastien Imperiale, Patrick Joly. Mathematical modeling of electromagnetic wave propagation in heterogeneous lossy coaxial cables with variable cross section. Applied Numerical Mathematics, 2013, 10.1016/j.apnum.2013.03.011 . hal-00875811

\section{HAL Id: hal-00875811 \\ https://inria.hal.science/hal-00875811}

Submitted on 22 Oct 2013

HAL is a multi-disciplinary open access archive for the deposit and dissemination of scientific research documents, whether they are published or not. The documents may come from teaching and research institutions in France or abroad, or from public or private research centers.
L'archive ouverte pluridisciplinaire HAL, est destinée au dépôt et à la diffusion de documents scientifiques de niveau recherche, publiés ou non, émanant des établissements d'enseignement et de recherche français ou étrangers, des laboratoires publics ou privés. 


\title{
Mathematical modeling of electromagnetic wave propagation in heterogeneous lossy coaxial cables with variable cross section
}

\author{
Sébastien Imperiale ${ }^{\mathrm{a}, \mathrm{b}}$, Patrick Joly ${ }^{\mathrm{b}}$ \\ ${ }^{a}$ INRIA Rocquencourt, POems, domaine de Voluceau, 78153 Le Chesnay, FRANCE \\ ${ }^{b}$ CEA, LIST, 91191 Gif-sur-Yvette CEDEX, FRANCE
}

\begin{abstract}
In this work, we focus on the time-domain simulation of the propagation of electromagnetic waves in non-homogeneous lossy coaxial cables. The full 3D Maxwell equations, that described the propagation of current and electric potential in such cables, are classically not tackled directly, but instead a 1D scalar model known as the telegraphist's model is used. We aim at justifying, by means of asymptotic analysis, a time-domain "homogenized" telegraphist's model. This model, which includes a non-local in time operator, is obtained via asymptotic analysis, for a lossy coaxial cable whose cross-section is not homogeneous.
\end{abstract}

Keywords: Coaxial cables, telegraphist's model, asymptotic analysis, Maxwell equations

2011 MSC: 35L05, 35A35, 73R05, 35A40

\section{Introduction and motivation. Objectives of the paper.}

Although of a rather fundamental nature, the present work has been motivated by an industrial application in the framework of a collaboration between the Project team POEMS of INRIA and the laboratory LIST of CEA Saclay. The general objective of this collaboration has been the numerical simulation of piezo-electric sensors used for non destructive testing

Email addresses: sebastien.imperiale@inria.fr (Sébastien Imperiale), patrick.joly@inria.fr (Patrick Joly) 
with ultrasonic waves. This led us to develop in [7] a specific finite element approach. One important aspect of the work is the modeling of the electromagnetic supply process : an electric field is generated by an electric generator and transmitted to the sensor via a coaxial electric cable: see Figure 1 (left and middle pictures).

The presence of the coaxial cable was neglected in [7]. However, as pointed
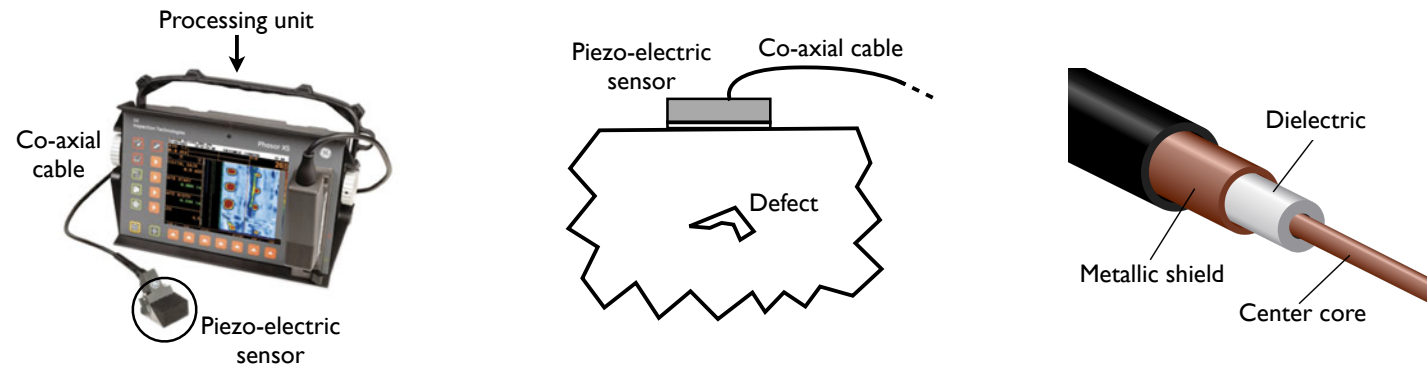

Figure 1: Left and center: Piezoelectric sensors, middle: coaxial cable.

out in [12], it can have an influence on the signal that is transmitted to the sensor as well on the recorded signal, especially if it is quite long, which can be the case in airplanes for instance. A cable being a structure whose transverse dimensions are much smaller than the longitudinal one, one would like to use a simplified 1D model. In such a situation, electrical engineers use the so-called telegraphist's equations where the electric unknowns are reduced to an electric potential $V\left(x_{3}, t\right)$ and an electric current $I\left(x_{3}, t\right)$, where $x_{3}$ denotes the abscissa along the cable and $t$ is time. The telegraphist's equations are generally used (see [13] for instance) in the case of a "perfect" coaxial cable: this corresponds to the case (illustrated on Figure 1, right picture) where the domain of propagation (namely the insulator) is a cylindrical annulus, filled with a homogeneous dielectric (possibly conducting) medium, which surrounds a perfectly conducting cylinder (the center core) and is surrounded by another cylindrical perfectly conducting medium (the metallic shield). The equations governing $V\left(x_{3}, t\right)$ and electric current $I\left(x_{3}, t\right)$ are

$$
\left\{\begin{array}{l}
C \frac{\partial V}{\partial t}+G V-\frac{\partial I}{\partial x_{3}}=0 \\
L \frac{\partial I}{\partial t}+R I-\frac{\partial V}{\partial x_{3}}=0
\end{array}\right.
$$


where the capacitance $C$, the inductance $L$, the conductance $G$ and the resistance $R$ can be expressed in terms of the interior and exterior radii $r_{1}$ and $r_{2}$ of the annulus and of the physical characteristics of the dielectric material, in particular the electric permittivity $\varepsilon$ and the magnetic permeability $\mu$. For instance, the coefficients $C$ and $L$ are given by

$$
C=2 \pi\left(\ln \frac{r_{2}}{r_{1}}\right)^{-1} \varepsilon, \quad L=\frac{1}{2 \pi}\left(\ln \frac{r_{2}}{r_{1}}\right) \mu .
$$

This telegraphist's equation can be obtained from a low frequency analysis of the equations for the fundamental mode of the cable (see [13] or [5]).

A natural question is: what does the approximate model (1) become in a more general and realistic situation namely when each cross section is heterogeneous (physical characteristics of the medium vary in the cross section) and the cable has a variable cross section and material properties along the cable (translation invariance and modal representations are lost).

This is the question ( perhaps considered as completely understood by electrical engineers, see for instance [6]) that has motivated us to look at Maxwell's equations in such "generalized" coaxial cables. Our objective was to derive a 1D effective model (let's say a generalized telegraphist's equation) via an asymptotic analysis with respect to the (small) transverse dimensions of the cable, in the spirit of what has been done in mechanics for the justification of plate, shell or beam models (see [2], [3], [14]) but has apparently not been done in electromagnetism. Above the intrinsic interest of such a study, we think that a profound understanding of the passage 3D-1D is a necessary preliminary step towards understanding the junction of several cables (see [9] or [10] for the acoustic case) or the connexion of a cable with another $2 \mathrm{D}$ or $3 \mathrm{D}$ structure (which we encounter, for instance, in the case of piezo-electric devices for the connexion between coaxial cables and thin electrodes).

In this first paper, we have chosen to restrict ourselves to formal asymptotic analysis and to the study in the time domain, of the effective 1D model. In section 2 , we describe the geometry of a coaxial cable and the family of problems, depending of a small parameter $\delta>0$, of which we make an asymptotic analysis, first in the frequency domain. In section 3, we explain the structure of the leading terms in the formal expansions. This leads us to the generalized telegraphist's equations (48) in the frequency domain, in which 
the effective (or homogenized) coefficients, which generalize $(C, L, R, G)$ in (1) are given by formulas (37)-(40). Section 4 concerns preparatory material before coming back to time domain. We establish in section 5 the generalized telegraphist's equations: the conjugated presence of electromagnetic losses and heterogeneity of the cross-section induces memory effects via time convolution terms in the equations.

\section{The mathematical model for coaxial cables}

To derive our effective model (see section 3), we consider a family of problems posed in domains, called cables, that depend on a small geometric parameter $\delta>0$ representing the transverse dimensions of the cables. Of course, a given cable corresponds to a given value of $\delta$ but the effective model will be constructed by an asymptotic analysis in $\delta$. These domains will be obtained by scaling with respect to appropriate transverse variables of a "normalized" reference cable. This normalized infinite $\Omega$ is defined as the union of its cross-sections (see Figure 2, left picture)

$$
\Omega=\cup_{z \in \mathbb{R}} S_{z}
$$

with $S_{z}$ a connected bounded and Lipschitz domain included in the plane $x_{3}=z$. In addition, we assume that for all $z \in \mathbb{R}, S_{z}$ includes the origin: $\mathbf{0} \in S_{z}$, which, in some sense, means that we consider straight cables. We also assume that $\partial \Omega$ is a smooth enough (Lipschitz) manifold along which the normal unit vector $\mathbf{n}$ satisfies

\section{Hypothesis 2.1.}

$$
\exists \nu \in] 0,1\left[\text { such that }\left|\mathbf{n} \cdot \mathbf{e}_{3}\right| \leq \nu<1, \quad \text { a. e. on } \partial \Omega .\right.
$$

Finally, we shall concentrate on the case of coaxial cables, namely cables whose cross-sections are not simply connected. For simplicity, we shall restrict ourselves to the case where each cross section contains only one hole (see Figure 2, middle picture)

Hypothesis 2.2. For all $z \in \mathbb{R}, S_{z}=\mathcal{O}_{z} \backslash \overline{T_{z}}$, where $\mathcal{O}_{z}$ and $T_{z}$ (the "hole", occupied by a perfect conductor) are simply connected, Lipschitz, open sets included in the plane $x_{3}=z$ with $\overline{T_{z}} \subset \subset \mathcal{O}_{z}$, and meas $T_{z} \geq a_{*}>0, \forall z \in \mathbb{R}$. In this case, the boundary of $S_{z}$ has two connected components, the exterior $\left(\partial S_{z}^{+}\right)$and the interior $\left(\partial S_{z}^{-}\right)$one $\partial S_{z}^{+} \equiv \partial \mathcal{O}_{z}, \quad \partial S_{z}^{-} \equiv \partial T_{z}$. 

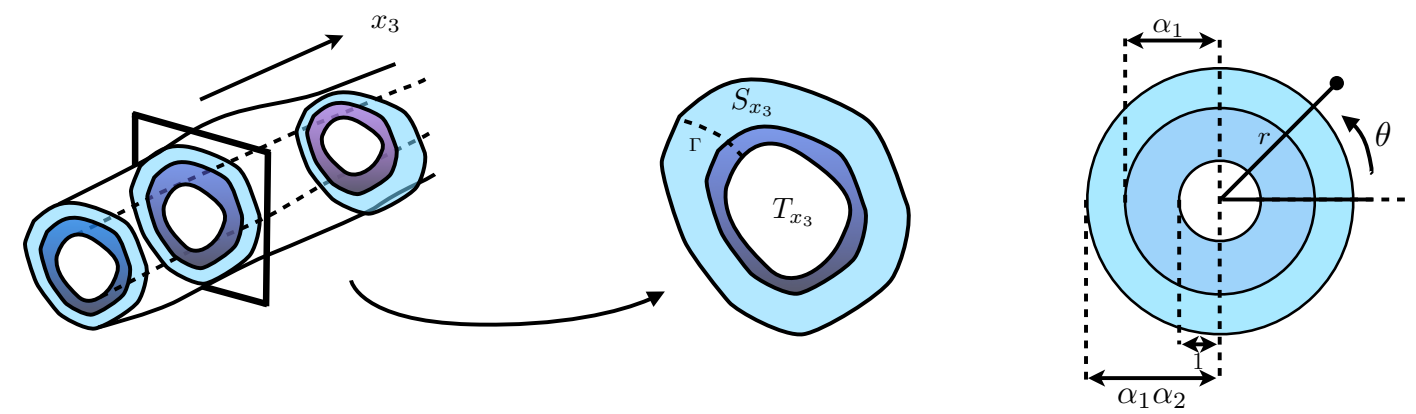

Figure 2: Left: domain of interest, center: slice of the domain, right: specific situation consider in section 5.3.

We consider a family of (thin) domains parametrized by a strictly positive scalar $\delta$ and defined by $\Omega^{\delta}=\mathcal{G}_{\delta}(\Omega)$, where $\mathcal{G}_{\delta}:\left(x_{1}, x_{2}, x_{3}\right) \longrightarrow\left(\delta x_{1}, \delta x_{2}, x_{3}\right)$.

Remark 2.1. Abuse of notation. In the sequel, we shall use systematically the passage from $\Omega$ to $\Omega^{\delta}$ via the change of variable $\mathcal{G}_{\delta}$. To avoid heavy notation, we shall use the same letters $(\mathbf{x}, \ldots)$ for the coordinates in $\Omega$ or $\Omega^{\delta}$, the meaning being fixed by the context. In the same way, we shall use the same notation for the differential operators in both variables (grad, rot,...).

Example 2.1. Consider the case where $\mathcal{O}_{z}$ and $T_{z}$ are star-like domains with respect to the origin. If $(r, \theta)$ are the polar coordinates in the $\left(x_{1}, x_{2}\right)$-plane, there exist two Lipschitz continuous functions $0<\rho_{-}\left(x_{3}, \theta\right)<\rho_{+}\left(x_{3}, \theta\right), 2 \pi$ periodic in $\theta$, such that

$$
\Omega=\left\{\left(x_{1}, x_{2}, x_{3}\right) \in \mathbb{R}^{3} / \rho_{-}\left(x_{3}, \theta\right)<r<\rho_{+}\left(x_{3}, \theta\right)\right\} .
$$

On $\partial \Omega^{\delta}$, it is useful to use a non unit outward normal vector $\mathbf{n}^{\delta}$ which has a unitary projection on the plane $\left(x_{1}, x_{2}\right)$. With some elementary geometry, $\mathbf{n}^{\delta}$ can be considered of the form

$$
\begin{aligned}
& \text { a. е. } \mathcal{G}_{\delta}(\mathbf{x}) \in \partial \Omega^{\delta}, \quad \mathbf{n}^{\delta} \circ \mathcal{G}_{\delta}(\mathbf{x})=\left(n_{1}(\mathbf{x}), n_{2}(\mathbf{x}), 0\right)^{t}+\delta g(\mathbf{x}) \mathbf{e}_{3}, \\
& \mathbf{n}_{T}=\left(n_{1}, n_{2}\right)^{t}: \partial \Omega \rightarrow \mathbb{R}^{2},\left|\mathbf{n}_{T}\right|=1, \quad g: \partial \Omega \rightarrow \mathbb{R} .
\end{aligned}
$$

Remark 2.2. In the case of example 2.1, along each part of the boundary

$$
n_{ \pm}(r, \theta)=\left(\cos \theta-\frac{\partial \rho_{ \pm}}{\partial \theta} \frac{\sin \theta}{\rho_{ \pm}}, \sin \theta+\frac{\partial \rho_{ \pm}}{\partial \theta} \frac{\cos \theta}{\rho_{ \pm}}\right)^{t}, \quad g_{ \pm}(r, \theta)=-\frac{\partial \rho_{ \pm}}{\partial x_{3}} .
$$


We shall assume that the permittivity $\varepsilon^{\delta}(\mathbf{x})$, the permeability $\mu^{\delta}(\mathbf{x})$, the electric and magnetic conductivity $\sigma_{e}^{\delta}(\mathbf{x})$ and $\sigma_{m}^{\delta}(\mathbf{x})$ are obtained by a scaling in $\mathbf{x}_{T}=\left(x_{1}, x_{2}\right)$, of fixed distributions over the domain $\Omega$ :

$$
\varepsilon^{\delta}=\varepsilon \circ \mathcal{G}_{\delta}^{-1}, \quad \mu^{\delta}=\mu \circ \mathcal{G}_{\delta}^{-1}, \quad \sigma_{e}^{\delta}=\sigma_{e} \circ \mathcal{G}_{\delta}^{-1}, \quad \sigma_{m}^{\delta}=\sigma_{m} \circ \mathcal{G}_{\delta}^{-1},
$$

where $\left(\varepsilon, \mu, \sigma_{e}, \sigma_{m}\right)$ satisfy the usual boundedness and positivity properties:

$$
\begin{aligned}
& 0<\varepsilon_{-} \leq \varepsilon(\mathbf{x}) \leq \varepsilon_{+}, \quad 0<\mu_{-} \leq \mu(\mathbf{x}) \leq \mu_{+}, \quad \mathbf{x} \in \Omega, \\
& 0 \leq \sigma_{e,-} \leq \sigma_{e}(\mathbf{x}) \leq \sigma_{e,+}, \quad 0 \leq \sigma_{m,-} \leq \sigma_{m}(\mathbf{x}) \leq \sigma_{m,+}, \quad \mathbf{x} \in \Omega \text {. }
\end{aligned}
$$

Example 2.2. Perfectly cylindrical cables. These correspond to the case where the problem is invariant under translation along the $x_{3}$ axis. This means that $\Omega$ is a cylindrical domain, $\Omega=S \times \mathbb{R}$, and that all coefficients are independent of $x_{3}: \varepsilon\left(x_{1}, x_{2}, x_{3}\right)=\varepsilon\left(x_{1}, x_{2}\right), \quad \mu\left(x_{1}, x_{2}, x_{3}\right)=\mu\left(x_{1}, x_{2}\right), \quad \ldots$

Example 2.3. Cables with homogeneous cross sections. These correspond to the situation where all coefficients only depend on the $x_{3}$ variable: $\varepsilon\left(x_{1}, x_{2}, x_{3}\right)=\varepsilon\left(x_{3}\right), \quad \mu\left(x_{1}, x_{2}, x_{3}\right)=\mu\left(x_{3}\right), \quad \ldots$

The equations that govern electromagnetic waves are Maxwell's equations

$$
\begin{cases}\varepsilon^{\delta} \frac{\partial}{\partial t} E^{\delta}+\sigma_{e}^{\delta} E^{\delta}-\nabla \times H^{\delta}=\mathbf{j}^{\delta}, & \text { in } \Omega^{\delta} \times \mathbb{R}^{+} \\ \mu^{\delta} \frac{\partial}{\partial t} H^{\delta}+\sigma_{m}^{\delta} H^{\delta}+\nabla \times E^{\delta}=\mathbf{0}, & \text { in } \Omega^{\delta} \times \mathbb{R}^{+}\end{cases}
$$

where $\boldsymbol{\nabla} \times$ is the 3D curl operator. We use perfectly conducting boundary conditions and zero initial conditions:

$$
\begin{gathered}
E^{\delta} \times \mathbf{n}^{\delta}=\mathbf{0} \quad \text { on } \partial \Omega^{\delta} \times \mathbb{R}^{+}, \\
E^{\delta}(\mathbf{x}, 0)=\mathbf{0}, \quad H^{\delta}(\mathbf{x}, 0)=\mathbf{0}, \quad \mathbf{x} \in \Omega^{\delta} .
\end{gathered}
$$

For the sake of simplicity, we shall assume that the source term, namely the current density $\mathbf{j}^{\delta}$ is such that

$$
\mathbf{j}^{\delta}=\mathbf{j} \circ \mathcal{G}_{\delta}^{-1}, \quad \mathbf{j}=\left(\mathbf{j}_{T}, 0\right)^{t}, \quad \mathbf{j}_{T}=\left(j_{1}, j_{2}\right)^{t}, \quad \frac{\partial j_{1}}{\partial x_{1}}+\frac{\partial j_{2}}{\partial x_{2}}=0, \quad \mathbf{x} \in \Omega
$$

It appears judicious to introduce the tangential components

$$
E_{T}^{\delta}=\left(E_{1}^{\delta}, E_{2}^{\delta}\right)^{t}, \quad H_{T}^{\delta}=\left(H_{1}^{\delta}, H_{2}^{\delta}\right)^{t}
$$


of the electric and magnetic fields as well as the longitudinal components of these fields: $E_{3}^{\delta}$ and $H_{3}^{\delta}$. We can rewrite the equations (4) with these new unknowns. To do so, we shall use, for all scalar functions $u$ and $2 \mathrm{D}$ vector fields $\mathbf{v}$ with two components $v_{1}$ and $v_{2}$, the following notations:

$$
\begin{aligned}
& \operatorname{grad} u \equiv\left(\frac{\partial u}{\partial x_{1}}, \frac{\partial u}{\partial x_{2}}\right)^{t}, \quad \operatorname{rot} u \equiv\left(\frac{\partial u}{\partial x_{2}},-\frac{\partial u}{\partial x_{1}}\right)^{t} \\
& \operatorname{div} \mathbf{v} \equiv \frac{\partial v_{1}}{\partial x_{1}}+\frac{\partial v_{2}}{\partial x_{2}}, \quad \operatorname{rot} \mathbf{v} \equiv \frac{\partial v_{2}}{\partial x_{1}}-\frac{\partial v_{1}}{\partial x_{2}}
\end{aligned}
$$

and, for any $\mathbf{v}=\left(v_{1}, v_{2}\right)$ and $\mathbf{u}=\left(u_{1}, u_{2}\right)$ (possibly complex valued),

$$
\mathbf{v} \cdot \mathbf{u} \equiv v_{1} u_{1}+v_{2} u_{2}, \quad \mathbf{v} \times \mathbf{u} \equiv v_{1} u_{2}-v_{2} u_{1}, \quad \mathbf{e}_{3} \times \mathbf{v} \equiv\left(-v_{2}, v_{1}\right)^{t},
$$

The reader will observe in particular that, for any scalar functions $u$ and $\widetilde{u}$,

$$
\begin{gathered}
\operatorname{rot} u \cdot \operatorname{grad} \widetilde{u}=-\operatorname{rot} \widetilde{u} \cdot \operatorname{grad} u \\
\mathbf{e}_{3} \times \operatorname{grad} u=-\operatorname{rot} u, \quad \operatorname{grad} u \times \mathbf{v}=-\mathbf{v} \cdot \operatorname{rot} u .
\end{gathered}
$$

We shall also use the Green's formula ( $\sigma_{x_{3}}$ is the lineic measure along $\partial S_{x_{3}}$ )

$$
(\operatorname{rot} u, \mathbf{v})_{L^{2}\left(S_{x_{3}}\right)}=(u, \operatorname{rot} \mathbf{v})_{L^{2}\left(S_{x_{3}}\right)}+\int_{\partial S_{x_{3}}} u \overline{\left(\mathbf{v} \times \mathbf{n}_{T}\right)} d \sigma_{x_{3}} .
$$

Remark 2.3. Formula (10) is valid for $(u, \mathbf{v})$ in $H^{1}\left(S_{x_{3}}\right) \times H\left(\operatorname{rot}, S_{x_{3}}\right)$ and the boundary integral must be (as usual) understood as a duality product.

The equations (4) can be rewritten in $\Omega^{\delta} \times \mathbb{R}^{+}$as

$$
\left\{\begin{array}{l}
\varepsilon^{\delta} \frac{\partial}{\partial t} E_{T}^{\delta}+\sigma_{e}^{\delta} E_{T}^{\delta}-\frac{\partial}{\partial x_{3}} \mathbf{e}_{3} \times H_{T}^{\delta}-\operatorname{rot} H_{3}^{\delta}=\mathbf{j}_{T} \circ \mathcal{G}_{\delta}^{-1} \\
\varepsilon^{\delta} \frac{\partial}{\partial t} E_{3}^{\delta}+\sigma_{e}^{\delta} E_{3}^{\delta}-\operatorname{rot} H_{T}^{\delta}=0 \\
\mu^{\delta} \frac{\partial}{\partial t} H_{T}^{\delta}+\sigma_{m}^{\delta} H_{T}^{\delta}+\frac{\partial}{\partial x_{3}} \mathbf{e}_{3} \times E_{T}^{\delta}+\operatorname{rot} E_{3}^{\delta}=\mathbf{0} \\
\mu^{\delta} \frac{\partial}{\partial t} H_{3}^{\delta}+\sigma_{m}^{\delta} H_{3}^{\delta}+\operatorname{rot} E_{T}^{\delta}=0 .
\end{array}\right.
$$

Moreover, using (2), the boundary conditions (5) on $\partial \Omega^{\delta} \times \mathbb{R}^{+}$become

$$
E_{T}^{\delta} \times\left(\mathbf{n}_{T} \circ \mathcal{G}_{\delta}^{-1}\right)=\mathbf{0}, \quad E_{3}^{\delta}\left(\mathbf{n}_{T} \circ \mathcal{G}_{\delta}^{-1}\right)-\delta\left(g \circ \mathcal{G}_{\delta}^{-1}\right) E_{T}^{\delta}=0
$$


Taking the divergence of the equations (11) and using (7), we get a set of "hidden" divergence equations in $\Omega^{\delta} \times \mathbb{R}^{+}$:

$$
\begin{aligned}
& \operatorname{div}\left(\varepsilon^{\delta} \frac{\partial}{\partial t} E_{T}^{\delta}+\sigma_{e}^{\delta} E_{T}^{\delta}\right)+\frac{\partial}{\partial x_{3}}\left(\varepsilon^{\delta} \frac{\partial}{\partial t} E_{3}^{\delta}+\sigma_{e}^{\delta} E_{3}^{\delta}\right)=0, \\
& \operatorname{div}\left(\mu^{\delta} \frac{\partial}{\partial t} H_{T}^{\delta}+\sigma_{m}^{\delta} H_{T}^{\delta}\right)+\frac{\partial}{\partial x_{3}}\left(\mu^{\delta} \frac{\partial}{\partial t} H_{3}^{\delta}+\sigma_{m}^{\delta} H_{3}^{\delta}\right)=0 .
\end{aligned}
$$

Moreover, from (14), (13) and (12) it is classical to derive an additional boundary condition for the magnetic field (see for instance [5]):

$$
H_{T}^{\delta} \cdot\left(\mathbf{n}_{T} \circ \mathcal{G}_{\delta}^{-1}\right)+\delta\left(g \circ \mathcal{G}_{\delta}^{-1}\right) H_{3}^{\delta}=0, \quad \text { on } \partial \Omega^{\delta} \times \mathbb{R}^{+} .
$$

We want to describe the behavior of $\left(E^{\delta}, H^{\delta}\right)$ when $\delta$ tends to 0 . For this, it is useful to apply a change of variables in order to work in a fixed geometry. Doing so, the parameter $\delta$ will appear in the coefficients of the governing equations. We introduce the rescaled fields $\left(\widetilde{E}_{T}^{\delta}, \widetilde{E}_{3}^{\delta}, \widetilde{H}_{T}^{\delta}, \widetilde{H}_{3}^{\delta}\right)$ defined by:

$$
E_{T}^{\delta}=\widetilde{E}_{T}^{\delta} \circ \mathcal{G}_{\delta}^{-1}, \quad E_{3}^{\delta}=\widetilde{E}_{3}^{\delta} \circ \mathcal{G}_{\delta}^{-1}, \quad H_{T}^{\delta}=\widetilde{H}_{T}^{\delta} \circ \mathcal{G}_{\delta}^{-1}, \quad H_{3}^{\delta}=\widetilde{H}_{3}^{\delta} \circ \mathcal{G}_{\delta}^{-1} .
$$

We can write from $(11,12)$ the equations for $\left(\widetilde{E}_{T}^{\delta}, \widetilde{E}_{3}^{\delta}, \widetilde{H}_{T}^{\delta}, \widetilde{H}_{3}^{\delta}\right)$ in the reference domain $\Omega$ using the abuse of notation explained in remark 2.1 (the $\delta^{-1}$ terms simply come from derivatives in $\left.\mathbf{x}_{T}=\left(x_{1}, x_{2}\right)\right)$ :

$$
\begin{cases}\varepsilon \frac{\partial}{\partial t} \widetilde{E}_{T}^{\delta}+\sigma_{e} \widetilde{E}_{T}^{\delta},-\frac{\partial}{\partial x_{3}} \mathbf{e}_{3} \times \widetilde{H}_{T}^{\delta}-\delta^{-1} \operatorname{rot} \widetilde{H}_{3}^{\delta}=\mathbf{j}_{T}, & \text { in } \Omega \times \mathbb{R}^{+}, \\ \varepsilon \frac{\partial}{\partial t} \widetilde{E}_{3}^{\delta}+\sigma_{e} \widetilde{E}_{3}^{\delta}-\delta^{-1} \operatorname{rot} \widetilde{H}_{T}^{\delta}=0, & \text { in } \Omega \times \mathbb{R}^{+} \\ \mu \frac{\partial}{\partial t} \widetilde{H}_{T}^{\delta}+\sigma_{m} \widetilde{H}_{T}^{\delta}+\frac{\partial}{\partial x_{3}} \mathbf{e}_{3} \times \widetilde{E}_{T}^{\delta}+\delta^{-1} \operatorname{rot} \widetilde{E}_{3}=\mathbf{0}, & \text { in } \Omega \times \mathbb{R}^{+} \\ \mu \frac{\partial}{\partial t} \widetilde{H}_{3}^{\delta}+\sigma_{m} \widetilde{H}_{3}^{\delta}+\delta^{-1} \operatorname{rot} \widetilde{E}_{T}^{\delta}=0, & \text { in } \Omega \times \mathbb{R}^{+}\end{cases}
$$

while from the divergence equations (13), we have, in $\Omega \times \mathbb{R}^{+}$

$$
\left\{\begin{array}{c}
\delta^{-1} \operatorname{div}\left(\varepsilon \frac{\partial}{\partial t} \widetilde{E}_{T}^{\delta}+\sigma_{e} \widetilde{E}_{T}^{\delta}\right)+\frac{\partial}{\partial x_{3}}\left(\varepsilon \frac{\partial}{\partial t} \widetilde{E}_{3}^{\delta}+\sigma_{e} \widetilde{E}_{3}^{\delta}\right)=0, \\
\delta^{-1} \operatorname{div}\left(\mu \frac{\partial}{\partial t} \widetilde{H}_{T}^{\delta}+\sigma_{m} \widetilde{H}_{T}^{\delta}\right)+\frac{\partial}{\partial x_{3}}\left(\mu \frac{\partial}{\partial t} \widetilde{H}_{3}^{\delta}+\sigma_{m} \widetilde{H}_{3}^{\delta}\right)=0
\end{array}\right.
$$

(14) and (15) are completed by zero initial conditions and boundary conditions easily deduced from $(12)$, on $\partial \Omega \times \mathbb{R}^{+}$, we find

$$
\widetilde{E}_{T}^{\delta} \times \mathbf{n}_{T}=\mathbf{0}, \quad \widetilde{E}_{3}^{\delta} \mathbf{n}_{T}-\delta g \widetilde{E}_{T}^{\delta}=0, \quad \widetilde{H}_{T}^{\delta} \cdot \mathbf{n}_{T}+\delta g \widetilde{H}_{3}^{\delta}=0 .
$$




\section{Formal derivation of the limit problem in the frequency domain}

It appears useful to work in the frequency domain, using the time Fourier transform of the unknown functions (all extended by 0 for negative time)

$$
\widetilde{E}_{T}^{\delta}(\mathbf{x}, t) \longrightarrow \widehat{E}_{T}^{\delta}(\mathbf{x}, \omega), \ldots \quad \text { and so on. }
$$

As we work with zero initial conditions, from (14), (15) and (16), we get:

$$
\begin{cases}\left(i \omega \varepsilon+\sigma_{e}\right) \widehat{E}_{T}^{\delta}-\frac{\partial}{\partial x_{3}} \mathbf{e}_{3} \times \widehat{H}_{T}^{\delta}-\delta^{-1} \operatorname{rot} \widehat{H}_{3}^{\delta}=\widehat{\mathbf{j}}_{T}, & \text { in } \Omega, \\ \left(i \omega \varepsilon+\sigma_{e}\right) \widehat{E}_{3}^{\delta}-\delta^{-1} \operatorname{rot} \widehat{H}_{T}^{\delta}=0, & \text { in } \Omega, \\ \left(i \omega \mu+\sigma_{m}\right) \widehat{H}_{T}^{\delta}+\frac{\partial}{\partial x_{3}} \mathbf{e}_{3} \times \widehat{E}_{T}^{\delta}+\delta^{-1} \operatorname{rot} \widehat{E}_{3}=\mathbf{0}, & \text { in } \Omega, \\ \left(i \omega \mu+\sigma_{m}\right) \widehat{H}_{3}^{\delta}+\delta^{-1} \operatorname{rot} \widehat{E}_{T}^{\delta}=0, & \text { in } \Omega,\end{cases}
$$

while from (15) we get

$$
\left\{\begin{array}{l}
\delta^{-1} \operatorname{div}\left(\left(i \omega \varepsilon+\sigma_{e}\right) \widehat{E}_{T}^{\delta}\right)+\frac{\partial}{\partial x_{3}}\left(\left(i \omega \varepsilon+\sigma_{e}\right) \widehat{E}_{3}^{\delta}\right)=0, \quad \text { in } \Omega \\
\delta^{-1} \operatorname{div}\left(\left(i \omega \mu+\sigma_{m}\right) \widehat{H}_{T}^{\delta}\right)+\frac{\partial}{\partial x_{3}}\left(\left(i \omega \mu+\sigma_{m}\right) \widehat{H}_{3}^{\delta}\right)=0, \quad \text { in } \Omega
\end{array}\right.
$$

The boundary conditions keep the same form

$$
\widehat{E}_{T}^{\delta} \times \mathbf{n}_{T}=\mathbf{0}, \quad \widehat{E}_{3}^{\delta} \mathbf{n}_{T}-\delta g \widehat{E}_{T}^{\delta}=0, \quad \widehat{H}_{T}^{\delta} \cdot \mathbf{n}_{T}+\delta g \widehat{H}_{3}^{\delta}=0, \quad \text { on } \partial \Omega .
$$

We look for a formal power series expansion in $\delta$ of the electromagnetic field

$$
\begin{array}{rlr}
\widehat{E}_{P}^{\delta}(\mathbf{x}, \omega) & =\widehat{E}_{P}^{0}(\mathbf{x}, \omega)+\delta \widehat{E}_{P}^{1}(\mathbf{x}, \omega)+\cdots, & P=3 \text { or } T, \\
\widehat{H}_{P}^{\delta}(\mathbf{x}, \omega)=\widehat{H}_{P}^{0}(\mathbf{x}, \omega)+\delta \widehat{H}_{P}^{1}(\mathbf{x}, \omega)+\cdots, & P=3 \text { or } T .
\end{array}
$$

We now adopt the usual methodology which consists in inserting (20) in (17) and identifying the series term by term, which begins by the $\delta^{-1}$ terms.

\subsection{Identification of the $\delta^{-1}$ terms : transverse polarization and "1D-structure" of the limit fields.}

From (17), (18) and (19) we deduce the equations

$$
\operatorname{rot} \widehat{H}_{3}^{0}=\operatorname{rot} \widehat{E}_{3}^{0}=\mathbf{0}, \quad \operatorname{rot} \widehat{H}_{T}^{0}=\operatorname{rot} \widehat{E}_{T}^{0}=0, \quad \text { in } \Omega,
$$




$$
\begin{gathered}
\operatorname{div}\left(\left(i \omega \varepsilon+\sigma_{e}\right) \widehat{E}_{T}^{0}\right)=0, \quad \operatorname{div}\left(\left(i \omega \mu+\sigma_{m}\right) \widehat{H}_{T}^{0}\right)=0, \quad \text { in } \Omega . \\
\widehat{E}_{T}^{0} \times \mathbf{n}_{T}=\mathbf{0}, \quad \widehat{E}_{3}^{0}=0, \quad \widehat{H}_{T}^{0} \cdot \mathbf{n}_{T}=0, \quad \text { on } \partial \Omega .
\end{gathered}
$$

We see that these equations only involve the leading terms of the expansions (20). However, they are not sufficient, to determine completely the (formal) limit field $\left(\widehat{E}^{0}, \widehat{H}^{0}\right)$. For this, we shall need also the $\delta^{0}$ terms. For the moment, we exploit equations (21) to (23).

First note that the first equation of (21) means that $\widehat{E}_{3}^{0}$ and $\widehat{H}_{3}^{0}$ are functions of $x_{3}$ only. Using (23), we deduce

$$
\widehat{E}_{3}^{0}(\mathbf{x}, \omega)=0, \quad \mathbf{x} \in \Omega .
$$

We shall show in section 3.2 that also $\widehat{H}_{3}^{0}=0$, that is: the limit field $\left(\widehat{E}^{0}, \widehat{H}^{0}\right)$ is transversely polarized.

Next, we are interested in the transverse electric field $\widehat{E}_{T}^{0}$. It is natural to introduce, for each $\left(x_{3}, \omega\right) \in \mathbb{R}^{2}$, the following space of $2 \mathrm{D}$ vector fields (below $\varepsilon \equiv \varepsilon\left(\cdot, x_{3}\right)$ and the same for $\left.\sigma_{e}\right)$ :

$$
\begin{gathered}
\mathcal{E}_{\text {stat }}\left(x_{3}, \omega\right) \equiv\left\{\mathbf{v} \in \mathbf{R}_{0}\left(x_{3}\right) / \operatorname{div}\left(\left(i \omega \varepsilon+\sigma_{e}\right) \mathbf{v}\right)=0, \mathbf{v} \times \mathbf{n}_{T}=0 \text { on } \partial S_{x_{3}}\right\} \\
\mathbf{R}_{0}\left(x_{3}\right) \equiv\left\{\mathbf{v} \in L^{2}\left(S_{x_{3}}\right)^{2} / \operatorname{rot} \mathbf{v}=0\right\}
\end{gathered}
$$

Indeed, equations (21) to (23) imply that,

$$
\forall\left(x_{3}, \omega\right) \in \mathbb{R}^{2}, \quad \widehat{E}_{T}^{0}\left(\cdot, x_{3}, \omega\right) \in \mathcal{E}_{\text {stat }}\left(x_{3}, \omega\right)
$$

A fundamental point is that, thanks to hypothesis $2.2, \mathcal{E}_{\text {stat }}\left(x_{3}, \omega\right)$ is a space of dimension 1. This is a consequence of a well known fundamental theorem on vector fields (see for instance [1],[5],[11]) that we state here in 2D:

Proposition 3.1. Let $\mathcal{O}$ be a bounded Lipschitz domain of $\mathbb{R}^{2}$. Let $\mathbf{v} \in$ $L^{2}(\mathcal{O})^{2}$, such that $\operatorname{rot} \mathbf{v}=0$. Assuming one of the following assumptions :

(i) $\mathcal{O}$ is simply connected, (ii) $\mathbf{v} \times \mathbf{n}_{T}=0$ on $\partial \mathcal{O}$,

there exists $\varphi \in H^{1}(\mathcal{O})$ such that $\mathbf{v}=\operatorname{grad} \varphi$ in $\mathcal{O}$. 
Lemma 3.1. For all $\left(x_{3}, \omega\right) \in \mathbb{R}^{2}$,

$$
\mathcal{E}_{\text {stat }}\left(x_{3}, \omega\right)=\operatorname{span}\left\{\operatorname{grad} \widehat{\varphi}_{s}\left(\cdot, x_{3}, \omega\right)\right\},
$$

where $\widehat{\varphi}_{s} \equiv \widehat{\varphi}_{s}\left(\cdot, x_{3}, \omega\right)$ is the unique function solution of the $2 D$ boundary value problem $\left(\left(x_{3}, \omega\right)\right.$ is a parameter $)$

$$
\left\{\begin{array}{l}
\operatorname{div}\left(\left(i \omega \varepsilon\left(\cdot, x_{3}\right)+\sigma_{e}\left(\cdot, x_{3}\right)\right) \operatorname{grad} \widehat{\varphi}_{s}\left(\cdot, x_{3}, \omega\right)\right)=0, \quad \text { in } S_{x_{3}}, \\
\widehat{\varphi}_{s}\left(\cdot, x_{3}, \omega\right)=0 \quad \text { on } \partial S_{x_{3}}^{+}, \quad \widehat{\varphi}_{s}\left(\cdot, x_{3}, \omega\right)=1 \quad \text { on } \partial S_{x_{3}}^{-} .
\end{array}\right.
$$

Proof This is a classical result that can be found in [5],[1],[11] in the case of real constant coefficients. For the reader's convenience, we adapt the proof to variable and complex coefficients. The existence and uniqueness of $\widehat{\varphi}_{s}\left(\cdot, x_{3}, \omega\right)$ solution of $(25)$ is a consequence of Lax-Milgram's theorem. We need to show now that every function $\mathbf{v} \in \mathbf{R}_{0}\left(S_{x_{3}}\right)$ such that

$$
\operatorname{div}\left(\left(i \omega \varepsilon\left(\cdot, x_{3}\right)+\sigma_{e}\left(\cdot, x_{3}\right)\right) \mathbf{v}\right)=0 \text { in } S_{x_{3}}, \quad \mathbf{v} \times \mathbf{n}_{T}=0 \text { on } \partial S_{x_{3}}
$$

is proportional to $\operatorname{grad} \widehat{\varphi}_{s}\left(\cdot, x_{3}, \omega\right)$. By proposition 3.1 (case (ii)), we know that $\mathbf{v}=\operatorname{grad} \varphi$ for some $\varphi \in H^{1}\left(S_{x_{3}}\right)$. From the second equality in (26), we deduce that $\operatorname{div}\left(\left(i \omega \varepsilon\left(\cdot, x_{3}\right)+\sigma_{e}\left(\cdot, x_{3}\right)\right) \operatorname{grad} \varphi\right)=0$ and from the third one that $\varphi$ is constant on each connected component of $\partial S_{x_{3}}$, which means that there exists $\left(\phi_{-}, \phi_{+}\right) \in \mathbb{C}^{2}$ such that $\varphi=\phi_{ \pm}$on $\partial S_{x_{3}}^{ \pm}$. From the uniqueness for the boundary value problem (25), we deduce that

$$
\varphi=\phi_{+}+\left(\phi_{-}-\phi_{+}\right) \widehat{\varphi}_{s}\left(\cdot, x_{3}, \omega\right), \quad \text { i. e. } \mathbf{v}=\left(\phi_{-}-\phi_{+}\right) \operatorname{grad} \widehat{\varphi}_{s}\left(\cdot, x_{3}, \omega\right)
$$

which concludes the proof.

From (24) and lemma 3.1, we deduce that, for each $\omega$, there exists a function $x_{3} \rightarrow \widehat{V}\left(x_{3}, \omega\right) \in \mathbb{C}$ such that:

$$
\widehat{E}_{T}^{0}\left(\cdot, x_{3}, \omega\right)=\widehat{V}\left(x_{3}, \omega\right) \operatorname{grad} \widehat{\varphi}_{s}\left(\cdot, x_{3}, \omega\right) .
$$

The formula (27) reveals the hidden "1D-structure" of the limit problem: the function $\widehat{\varphi}_{s}\left(\cdot, x_{3}, \omega\right)$ can be pre-computed by solving $2 D$-problems in each cross section (independently of the source, $x_{3}$ and $\omega$ are simply parameters) and the only unknown is the $1 \mathrm{D}$ function $V\left(x_{3}, \omega\right)$. 
Remark 3.1. Two particular cases deserve some comments:

- In the perfectly cylindrical case of Example 2.2, the function $\widehat{\varphi}_{s}(\mathbf{x}, \omega)$ is obviously independent of $x_{3}: \widehat{\varphi}_{s}(\mathbf{x}, \omega) \equiv \widehat{\varphi}_{s}\left(\mathbf{x}_{T}, \omega\right)$ and (27) becomes

$$
\widehat{E}_{T}^{0}\left(\cdot, x_{3}, \omega\right)=\widehat{V}\left(x_{3}, \omega\right) \operatorname{grad} \widehat{\varphi}_{s}(\cdot, \omega) .
$$

In other words, at the limit $\delta \rightarrow 0$, one has separation of variables in $\left(x_{1}, x_{2}\right)$ and $x_{3}$.

- If, in each cross section, $\varepsilon$ and $\sigma_{e}$ are proportional, that is to say if there exists a function $\alpha\left(x_{3}\right)$ such that

$$
\sigma_{e}\left(\cdot, x_{3}\right)=\alpha\left(x_{3}\right) \varepsilon\left(\cdot, x_{3}\right), \quad \text { in } \Omega,
$$

one sees that the function $\widehat{\varphi}_{s}\left(\cdot, x_{3}, \omega\right)$ is real valued and independent of $\omega: \widehat{\varphi}_{s}\left(\cdot, x_{3}, \omega\right) \equiv \varphi_{s}^{\infty}\left(\cdot, x_{3}\right)$ solution of

$$
\left\{\begin{array}{l}
\operatorname{div}\left(\varepsilon\left(\cdot, x_{3}\right) \operatorname{grad} \varphi_{s}^{\infty}\left(\cdot, x_{3}\right)\right)=0, \quad \text { in } S_{x_{3}}, \\
\varphi_{s}^{\infty}\left(\cdot, x_{3}\right)=0 \quad \text { on } \partial S_{x_{3}}^{+}, \quad \varphi_{s}^{\infty}\left(\cdot, x_{3}\right)=1 \quad \text { on } \partial S_{x_{3}}^{-} .
\end{array}\right.
$$

Next, we look at the transverse magnetic field $\widehat{H}_{T}^{0}$. Looking at equations (21) to (23), we are led to introduce, for each $\left(x_{3}, \omega\right) \in \mathbb{R}^{2}$, the following space of $2 \mathrm{D}$ vector fields (below $\mu$ holds for $\mu\left(\cdot, x_{3}\right)$ and the same for $\sigma_{m}$ ):

$\mathcal{H}_{\text {stat }}\left(x_{3}, \omega\right) \equiv\left\{\mathbf{v} \in \mathbf{R}_{0}\left(S_{x_{3}}\right) / \operatorname{div}\left(\left(i \omega \mu+\sigma_{m}\right) \mathbf{v}\right)=0, \mathbf{v} \cdot \mathbf{n}_{T}=0\right.$ on $\left.\partial S_{x_{3}}\right\}$

so that equations (21) to (23) imply that

$$
\forall\left(x_{3}, \omega\right) \in \mathbb{R}^{2}, \quad \widehat{H}_{T}^{0}\left(\cdot, x_{3}, \omega\right) \in \mathcal{H}_{\text {stat }}\left(x_{3}, \omega\right)
$$

We observe that, $\mathcal{H}_{\text {stat }}\left(x_{3}, \omega\right)$ only differs from $\mathcal{E}_{\text {stat }}\left(x_{3}, \omega\right)$ by the boundary condition on $\partial S_{x_{3}}$. To describe this space, it is useful to introduce an artificial "cut", namely a (possibly curved) line $\Gamma \subset S_{x_{3}}$ (see Figure 2, right picture) that connects the interior boundary $\partial S_{x_{3}}^{-}$to the exterior one $\partial S_{x_{3}}^{+}$in such a way that the domain

$$
S_{x_{3}}^{\Gamma} \equiv S_{x_{3}} \backslash \Gamma \quad \text { is simply connected. }
$$


We shall define an orientation of $\Gamma$ by introducing along $\Gamma$ the unit tangent vector $\tau$ that points from the exterior boundary $\partial S_{x_{3}}^{+}$towards the interior boundary $\partial S_{x_{3}}^{-}$. Accordingly we define along $\Gamma$ the unit normal vector:

$$
\mathbf{n}_{T}:=\mathbf{e}_{3} \times \boldsymbol{\tau}
$$

We also need to define the jump across $\Gamma$, denoted $[u]_{\Gamma}$, of a function $u$ which is sufficiently smooth from each side of $\Gamma$. This is done accordingly with the orientation of the vector $\mathbf{n}_{T}$, namely:

$$
\forall \mathbf{x}_{T} \in \Gamma, \quad[u]_{\Gamma}\left(\mathbf{x}_{T}\right) \equiv \lim _{\eta \searrow 0}\left(u\left(\mathbf{x}_{T}+\eta \mathbf{n}_{T}\right)-u\left(\mathbf{x}_{T}-\eta \mathbf{n}_{T}\right)\right)
$$

We shall also define by $\tau$ the curvilinear abscissa along $\Gamma$ which is coherent with the orientation of $\Gamma$ with the tangent vector $\boldsymbol{\tau}$. Doing so, one has

$$
\operatorname{grad} u \times \mathbf{n}_{T}=-\frac{\partial u}{\partial \tau}
$$

Finally, if $\psi \in H^{1}\left(S_{x_{3}}^{\Gamma}\right)$, we denote $\widetilde{\operatorname{grad}} \psi$ the vector in $L^{2}\left(S_{x_{3}}\right)^{2}$ such that

$$
\widetilde{\operatorname{grad}} \psi=\operatorname{grad} \psi \text { in } S_{x_{3}}^{\Gamma} \quad\left(\text { but } \widetilde{\operatorname{grad}} \psi \neq \operatorname{grad} \psi \text { in } S_{x_{3}} !\right)
$$

moreover we define $\widetilde{\operatorname{rot}} \psi=-\mathbf{e}_{3} \times \widetilde{\operatorname{grad}} \psi$. The description of $\mathcal{H}_{\text {stat }}\left(x_{3}, \omega\right)$ will be based on the following result.

Lemma 3.2. For any $\left(x_{3}, \omega\right)$, there is a unique $\widehat{\psi}_{s}\left(\cdot, x_{3}, \omega\right)$ so that

$$
\left\{\begin{array}{l}
\widehat{\psi}_{s}\left(\cdot, x_{3}, \omega\right) \in H^{1}\left(S_{x_{3}}^{\Gamma}\right) \text { and } \int_{S_{x_{3}}} \widehat{\psi}_{s}\left(\mathbf{x}_{T}, x_{3}, \omega\right) d \mathbf{x}_{T}=0 \\
\operatorname{div}\left(\left(i \omega \mu\left(\cdot, x_{3}\right)+\sigma_{m}\left(\cdot, x_{3}\right)\right) \operatorname{grad} \widehat{\psi}_{s}\left(\cdot, x_{3}, \omega\right)\right)=0, \quad \text { in } S_{x_{3}}^{\Gamma}, \\
{\left[\left(i \omega \mu\left(\cdot, x_{3}\right)+\sigma_{m}\left(\cdot, x_{3}\right)\right) \frac{\partial \widehat{\psi}_{s}\left(\cdot, x_{3}, \omega\right)}{\partial n}\right]_{\Gamma}=0, \quad\left[\widehat{\psi}_{s}\left(\cdot, x_{3}, \omega\right)\right]_{\Gamma}=1} \\
\frac{\partial \widehat{\psi}_{s}}{\partial n}\left(\cdot, x_{3}, \omega\right)=0, \quad \text { on } \partial S_{x_{3}} .
\end{array}\right.
$$

Proof This result is a slight variant of a result from [5] (theorem 3, p. 24). We give a proof for the reader's convenience. One easily checks that the weak 
formulation of (30) is (denoting $\widehat{\psi}_{s}=\psi_{s}\left(x_{3}, \omega\right), \mu=\mu\left(x_{3}, \omega\right)$, etc ...)

$$
\left\{\begin{array}{l}
\text { Find } \widehat{\psi}_{s} \in H^{1}\left(S_{x_{3}}^{\Gamma}\right) \text { such that }\left[\widehat{\psi}_{s}\right]_{\Gamma}=1, \int_{S_{x_{3}}} \widehat{\psi}_{s} d \mathbf{x}_{T}=0 \text { and } \\
\forall \psi \in H^{1}\left(S_{x_{3}}\right), \quad \int_{S_{x_{3}}}\left(i \omega \mu+\sigma_{m}\right) \operatorname{grad} \widehat{\psi}_{s} \cdot \operatorname{grad} \psi d \mathbf{x}_{T}=0
\end{array}\right.
$$

Writing $\widehat{\psi}_{s}=\psi^{*}+\widehat{\theta}_{s}$ for a given $\psi^{*} \in H^{1}\left(S_{x_{3}}^{\Gamma}\right)$ such that $\left[\psi^{*}\right]_{\Gamma}=1$ and with mean value 0 , the new unknown $\widehat{\theta}_{s}$ is solution of:

$$
\left\{\begin{aligned}
& \text { Find } \widehat{\theta}_{s} \in H^{1}\left(S_{x_{3}}\right) \text { such that } \int_{S_{x_{3}}} \widehat{\theta}_{s} d \mathbf{x}_{T}=0 \text { and } \\
& \forall \psi \in H^{1}\left(S_{x_{3}}\right), \int_{S_{x_{3}}}\left(i \omega \mu+\sigma_{m}\right) \operatorname{grad} \widehat{\theta}_{s} \cdot \operatorname{grad} \psi d \mathbf{x}_{T}= \\
&-\int_{S_{x_{3}}}\left(i \omega \mu+\sigma_{m}\right)\left(\cdot, x_{3}\right) \widetilde{\operatorname{grad}} \psi^{*} \cdot \operatorname{grad} \psi d \mathbf{x}_{T} .
\end{aligned}\right.
$$

One concludes using Poincaré-Wirtinger inequality $\left(S_{x_{3}}\right.$ is connected) and Lax-Milgram's lemma.

Lemma 3.3. For all $\left(x_{3}, \omega\right) \in \mathbb{R}^{2}$,

$$
\mathcal{H}_{\text {stat }}\left(x_{3}, \omega\right)=\operatorname{span}\left\{\widetilde{\operatorname{grad}} \widehat{\psi}_{s}\left(\cdot, x_{3}, \omega\right)\right\} .
$$

Proof We want to show that any function $\mathbf{v} \in \mathbf{R}_{0}\left(S_{x_{3}}\right)$ such that

$$
\operatorname{div}\left(\left(i \omega \mu\left(\cdot, x_{3}\right)+\sigma_{m}\left(\cdot, x_{3}\right)\right) \mathbf{v}\right)=0 \text { in } S_{x_{3}}, \quad \mathbf{v} \cdot \mathbf{n}_{T}=0 \text { on } \partial S_{x_{3}}
$$

is proportional to $\widetilde{\operatorname{grad}} \widehat{\psi}_{s}\left(\cdot, x_{3}, \omega\right)$. Since $S_{x_{3}}^{\Gamma}$ is by construction simply connected, we can apply lemma 3.1 (case (i)) to deduce that $\mathbf{v}=\operatorname{grad} \psi$ for some $\psi \in H^{1}\left(S_{x_{3}}^{\Gamma}\right)$. Moreover, $\operatorname{rot} \mathbf{v}=0$ in $S_{x_{3}}$, we know that the tangential component of $\mathbf{v}$ does not jump across $\Gamma$, namely the jump of $\psi$ is constant along $\Gamma$ : there exists a scalar $\phi \in \mathbb{R}$ such that $[\psi]_{\Gamma}=\phi$. As $\left[\widehat{\psi}_{s}\right]=1$ and as $\operatorname{grad} \widehat{\psi}_{s}$ satisfies $(31)$ we have $\mathbf{v}=\phi \operatorname{grad} \widehat{\psi}_{s}$.

As we have defined $\widehat{V}\left(x_{3}, \omega\right)$, we choose to define $\widehat{I}\left(x_{3}, \omega\right)$ by

$$
\widehat{H}_{T}^{0}\left(\cdot, x_{3}, \omega\right)=\widehat{I}\left(x_{3}, \omega\right) \widetilde{\operatorname{grad}} \widehat{\psi}_{s}\left(\cdot, x_{3}, \omega\right) .
$$


Remark 3.2. Analogously to remark 3.1

- In the perfectly cylindrical case of Example 2.2, the function $\widehat{\psi}_{s}\left(\cdot, x_{3}, \omega\right)$ is obviously independent of $x_{3}: \widehat{\psi}_{s}\left(\cdot, x_{3}, \omega\right) \equiv \widehat{\psi}_{s}(\cdot, \omega)$ and one has asymptotic separation of variables when $\delta \rightarrow 0$ :

$$
\widehat{H}_{T}^{0}\left(\cdot, x_{3}, \omega\right)=\widehat{I}\left(x_{3}, \omega\right) \widetilde{\operatorname{grad}} \widehat{\psi}_{s}(\cdot, \omega) .
$$

- If, in each cross section $\mu$ and $\sigma_{m}$ are proportional (in the sense of (28)), the function $\widehat{\psi}_{s}\left(\cdot, x_{3}, \omega\right)$ is real valued and independent of $\omega$ : $\widehat{\psi}_{s}\left(\cdot, x_{3}, \omega\right) \equiv \psi_{s}^{\infty}\left(\cdot, x_{3}\right)$ solution of

$$
\left\{\begin{array}{l}
\operatorname{div}\left(\mu\left(\cdot, x_{3}\right) \operatorname{grad} \psi_{s}^{\infty}\right)=0, \quad \text { in } S_{x_{3}}^{\Gamma}, \quad\left[\psi_{s}^{\infty}\right]_{\Gamma}=1 \\
\frac{\partial \psi_{s}^{\infty}\left(\cdot, x_{3}\right)}{\partial n}=0, \quad \text { on } \partial S_{x_{3}}, \quad\left[\mu\left(\cdot, x_{3}\right) \frac{\partial \psi_{s}^{\infty}}{\partial n}\right]_{\Gamma}=0
\end{array}\right.
$$

Remark 3.3. In the case of cables with homogeneous cross-sections (Example 2.3), there is a particularly simple relationship between the two spaces $\mathcal{E}_{\text {stat }}\left(x_{3}\right) \equiv \mathcal{E}_{\text {stat }}\left(x_{3}, \omega\right)$ and $\mathcal{H}_{\text {stat }}\left(x_{3}\right) \equiv \mathcal{H}_{\text {stat }}\left(x_{3}, \omega\right)$ (note that they are independent of $\omega$ by remarks 3.1 and 3.2). The vector fields of one space are obtained by rotating the ones of the other space by $\pi / 2$

$$
\mathcal{H}_{\text {stat }}\left(x_{3}\right)=\left\{\mathbf{e}_{\mathbf{3}} \times \mathbf{v}, / \mathbf{v} \in \mathcal{E}_{\text {stat }}\left(x_{3}\right)\right\} .
$$

It remains, in order to determine the formal limit fields $\left(\widehat{E}_{T}^{0}, \widehat{H}_{T}^{0}\right)$, to find the equations for $\widehat{V}$ and $\widehat{I}$. This is the object of the next section.

3.2. Identification of the $\delta^{0}$ terms : derivation of the $1 D$ effective model.

Using the hypothesis (20) and identification of the term in power of $\delta^{0}$ in (14), we obtain the equations :

$$
\begin{cases}\left(i \varepsilon \omega+\sigma_{e}\right) \widehat{E}_{T}^{0}-\frac{\partial}{\partial x_{3}} \mathbf{e}_{3} \times \widehat{H}_{T}^{0}-\operatorname{rot} \widehat{H}_{3}^{1}=\widehat{\mathbf{j}}_{T}, & \text { in } \Omega, \\ \left(i \varepsilon \omega+\sigma_{e}\right) \widehat{E}_{3}^{0}-\operatorname{rot} \widehat{H}_{T}^{1}=0, & \text { in } \Omega, \\ \left(i \mu \omega+\sigma_{m}\right) \widehat{H}_{T}^{0}+\frac{\partial}{\partial x_{3}} \mathbf{e}_{3} \times \widehat{E}_{T}^{0}+\operatorname{rot} \widehat{E}_{3}^{1}=\mathbf{0}, & \text { in } \Omega, \\ \left(i \mu \omega+\sigma_{m}\right) \widehat{H}_{3}^{0}+\operatorname{rot} \widehat{E}_{T}^{1}=0, & \text { in } \Omega .\end{cases}
$$


These equations are completed by boundary conditions for $\widehat{E}_{T}^{1}$ and $\widehat{E}_{3}^{1}$ :

$$
\widehat{E}_{T}^{1} \times \mathbf{n}_{T}=0, \quad \widehat{E}_{3}^{1} \mathbf{n}_{T}-g \widehat{E}_{T}^{0}=0, \quad \text { on } \partial \Omega,
$$

that are obtained by identification of terms in $\delta$ in (19).

We are now in position to prove that, as announced in the previous section, the longitudinal magnetic field $\widehat{H}_{3}^{0}$ vanishes. We already know (see section 3.1) that this longitudinal field does not depend on $x_{1}$ and $x_{2}$. Integrating the last equation of (34) over $S_{x_{3}}$, we get by Green's formula

$$
\widehat{H}_{3}^{0}\left(x_{3}, \omega\right) \int_{S_{x_{3}}}\left(i \omega \mu+\sigma_{m}\right)\left(\mathbf{x}_{T}, x_{3}\right) d \mathbf{x}_{T}=-\int_{\partial S_{x_{3}}} \widehat{E}_{T}^{1} \times \mathbf{n}_{T} d \sigma_{x_{3}}=0
$$

where we have used (35). Since $\int_{S_{x_{3}}}\left(i \omega \mu+\sigma_{m}\right)\left(\mathbf{x}_{T}, x_{3}\right) d \mathbf{x}_{T} \neq 0$,

$$
\forall \omega \in \mathbb{R}, \quad \widehat{H}_{3}^{0}(\mathbf{x}, \omega)=0, \quad \mathbf{x} \in \Omega .
$$

The remaining of this section will be rather formal and justified only under appropriate regularity assumptions about the data of the problem (source, coefficients). However everything can be made rigorous by proceeding in the weak sense (an example of which is given in the proof of (iii) lemma 3.4) and using appropriate regularization processes. Details can be found in [8].

To get the equations in $\widehat{V}$ and $\widehat{I}$ defined by $(27,32)$, we use the first and third equations of (34). These equations relate $\widehat{E}_{T}^{0}$ and $\widehat{H}_{T}^{0}$ to $\widehat{E}_{3}^{1}$ and $\widehat{H}_{3}^{1}$ which are not known for the moment. To get rid of them, we take, in each $S_{x_{3}}$, the $L^{2}$ scalar product of these two equations respectively with $\operatorname{grad} \widehat{\varphi}_{s}(\mathbf{x}, \omega)$ and $\operatorname{grad} \widehat{\psi}_{s}(\mathbf{x}, \omega)$. We obtain (modulo some obvious notation, in particular we write $\widehat{\varphi}_{s}$ instead of $\widehat{\varphi}_{s}(\mathbf{x}, \omega)$, etc $\left.\ldots\right)$, for all $x_{3} \in \mathbb{R}$.

$$
\left\{\begin{aligned}
(i \omega \widehat{C}+\widehat{G}) \widehat{V} & -\left(\frac{\partial}{\partial x_{3}} \mathbf{e}_{3} \times \widehat{H}_{T}^{0}, \operatorname{grad} \widehat{\varphi}_{s}\right)_{L^{2}\left(S_{x_{3}}\right)} \\
& -\left(\operatorname{rot} \widehat{H}_{3}^{1}, \operatorname{grad} \widehat{\varphi}_{s}\right)_{L^{2}\left(S_{x_{3}}\right)}=\left(\widehat{\mathbf{j}}_{T}, \operatorname{grad} \widehat{\varphi}_{s}\right)_{L^{2}\left(S_{x_{3}}\right)}, \\
(i \omega \widehat{L}+\widehat{R}) \widehat{I} & +\left(\frac{\partial}{\partial x_{3}} \mathbf{e}_{3} \times \widehat{E}_{T}^{0}, \widetilde{\operatorname{grad}} \widehat{\psi}_{s}\right)_{L^{2}\left(S_{x_{3}}\right)} \\
+ & \left(\operatorname{rot} \widehat{E}_{3}^{1}, \widetilde{\operatorname{grad}} \widehat{\psi}_{s}\right)_{L^{2}\left(S_{x_{3}}\right)}=0,
\end{aligned}\right.
$$


where the homogenized coefficients $(\widehat{C}, \widehat{L}, \widehat{G}, \widehat{R})$ depend on $x_{3}$ and $\omega$ :

$$
\begin{aligned}
\widehat{C}\left(x_{3}, \omega\right) & \equiv \int_{S_{x_{3}}} \varepsilon\left(\mathbf{x}_{T}, x_{3}\right)\left|\operatorname{grad} \widehat{\varphi}_{s}\left(\mathbf{x}_{T}, x_{3}, \omega\right)\right|^{2} d \mathbf{x}_{T} \\
\widehat{L}\left(x_{3}, \omega\right) & \equiv \int_{S_{x_{3}}} \mu\left(\mathbf{x}_{T}, x_{3}\right)\left|\widetilde{\operatorname{grad}} \widehat{\psi}_{s}\left(\mathbf{x}_{T}, x_{3}, \omega\right)\right|^{2} d \mathbf{x}_{T} \\
\widehat{G}\left(x_{3}, \omega\right) & \equiv \int_{S_{x_{3}}} \sigma_{e}\left(\mathbf{x}_{T}, x_{3}\right)\left|\operatorname{grad} \widehat{\varphi}_{s}\left(\mathbf{x}_{T}, x_{3}, \omega\right)\right|^{2} d \mathbf{x}_{T} \\
\widehat{R}\left(x_{3}, \omega\right) & \equiv \int_{S_{x_{3}}} \sigma_{m}\left(\mathbf{x}_{T}, x_{3}\right)\left|\widetilde{\operatorname{grad}} \widehat{\psi}_{s}\left(\mathbf{x}_{T}, x_{3}, \omega\right)\right|^{2} d \mathbf{x}_{T}
\end{aligned}
$$

Next, we treat the remaining terms in the first equation of (36). Using (32) together with (9), we have

$$
\begin{aligned}
\left(\frac{\partial}{\partial x_{3}} \mathbf{e}_{3} \times \widehat{H}_{T}^{0}, \widetilde{\operatorname{grad}} \widehat{\varphi}_{s}\right)_{L^{2}\left(S_{x_{3}}\right)}= & -\left(\widetilde{\operatorname{rot}} \widehat{\psi}_{s}, \operatorname{grad} \widehat{\varphi}_{s}\right)_{L^{2}\left(S_{x_{3}}\right)} \frac{\partial \widehat{I}}{\partial x_{3}} \\
& -\left(\frac{\partial}{\partial x_{3}} \widetilde{\operatorname{rot}} \widehat{\psi}_{s}, \operatorname{grad} \widehat{\varphi}_{s}\right)_{L^{2}\left(S_{x_{3}}\right)} \widehat{I} .
\end{aligned}
$$

Moreover, using Green's formula (10), we have

$$
\left(\operatorname{rot} \widehat{H}_{3}^{1}, \operatorname{grad} \widehat{\varphi}_{s}\right)_{L^{2}\left(S_{x_{3}}\right)}=\int_{\partial S_{x_{3}}} \widehat{H}_{3}^{1} \overline{\left(\operatorname{grad} \widehat{\varphi}_{s} \times \mathbf{n}_{T}\right)} d \sigma_{x_{3}}=0
$$

since $\operatorname{grad} \widehat{\varphi}_{s} \times \mathbf{n}_{T}=0$ along $\partial S_{x_{3}}$ and $\operatorname{rot} \operatorname{grad} \widehat{\varphi}_{s}=0$. In the same way, for the second equation of (36), using (27), we get

$$
\begin{aligned}
\left(\frac{\partial}{\partial x_{3}} \mathbf{e}_{3} \times \widehat{E}_{T}^{0}, \widetilde{\operatorname{grad}} \widehat{\psi}_{s}\right)_{L^{2}\left(S_{x_{3}}\right)} & =-\left(\operatorname{rot} \widehat{\varphi}_{s}, \widetilde{\operatorname{grad}} \widehat{\psi}_{s}\right)_{L^{2}\left(S_{x_{3}}\right)} \frac{\partial \widehat{V}}{\partial x_{3}} \\
& -\left(\frac{\partial}{\partial x_{3}} \operatorname{rot} \widehat{\varphi}_{s}, \widetilde{\operatorname{grad}} \widehat{\psi}_{s}\right)_{L^{2}\left(S_{x_{3}}\right)} \widehat{V} .
\end{aligned}
$$

On the other hand, by Green's formula

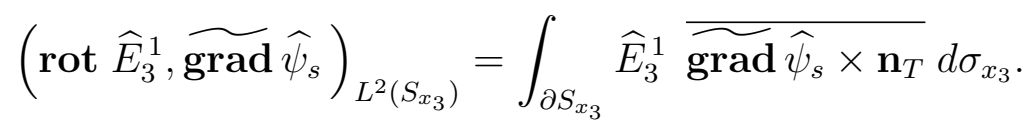


Using (9) and the second equation of (35), we get rid of $\widehat{E}_{3}^{1}$ and obtain

$$
\begin{aligned}
\left(\operatorname{rot} \widehat{E}_{3}^{1}, \widetilde{\operatorname{grad}} \widehat{\psi}_{s}\right)_{L^{2}\left(S_{x_{3}}\right)} & =-\int_{\partial S_{x_{3}}} g \widehat{E}_{T}^{0} \cdot \widetilde{\operatorname{rot}} \widehat{\psi}_{s} d \sigma_{x_{3}} \\
& =-\left(\int_{\partial S_{x_{3}}} g \operatorname{grad} \widehat{\varphi}_{s} \cdot \overline{\widetilde{\operatorname{rot}} \widehat{\psi}_{s}}\right) \widehat{V} d \sigma_{x_{3}}
\end{aligned}
$$

where we have used again (27).

Finally, we obtain formally the equations governing the variations of $\widehat{V}$ and $\widehat{I}$ by substituting $(41,42,43,44)$ into $(36)$ :

$$
\left\{\begin{array}{c}
(i \omega \widehat{C}+\widehat{G}) \widehat{V}+\left(\widetilde{\operatorname{rot}} \widehat{\psi}_{s}, \operatorname{grad} \widehat{\varphi}_{s}\right)_{L^{2}\left(S_{x_{3}}\right)} \frac{\partial \widehat{I}}{\partial x_{3}} \\
+\left(\frac{\partial}{\partial x_{3}} \widetilde{\operatorname{rot}} \widehat{\psi}_{s}, \operatorname{grad} \widehat{\varphi}_{s}\right)_{L^{2}\left(S_{x_{3}}\right)} \widehat{I}=\left(\widehat{\mathbf{j}}_{T}, \operatorname{grad} \widehat{\varphi}_{s}\right)_{L^{2}\left(S_{x_{3}}\right)} \\
(i \omega \widehat{L}+\widehat{R}) \widehat{I}-\left(\operatorname{rot} \widehat{\varphi}_{s}, \widetilde{\operatorname{grad}} \widehat{\psi}_{s}\right)_{L^{2}\left(S_{x_{3}}\right)} \frac{\partial \widehat{V}}{\partial x_{3}} \\
-\left(\frac{\partial}{\partial x_{3}} \operatorname{rot} \widehat{\varphi}_{s}, \widetilde{\operatorname{grad}} \widehat{\psi}_{s}\right)_{L^{2}\left(S_{x_{3}}\right)} \widehat{V} \\
-\left(\int_{\partial S_{x_{3}}} g \operatorname{grad} \widehat{\varphi}_{s} \cdot \widetilde{\operatorname{grad} \widehat{\psi}_{s}} d \sigma_{x_{3}}\right) \widehat{V}=0 .
\end{array}\right.
$$

We obtain further simplification thanks to the following lemma:

Lemma 3.4. One has the following identities (writing again $\widehat{\varphi}_{s}$ instead of $\widehat{\varphi}_{s}\left(x_{3}, \omega\right)$, etc $\left.\ldots\right)$

(i) $\left(\widetilde{\operatorname{rot}} \widehat{\psi}_{s}, \operatorname{grad} \widehat{\varphi}_{s}\right)_{L^{2}\left(S_{x_{3}}\right)}=-\left(\operatorname{rot} \widehat{\varphi}_{s}, \widetilde{\operatorname{grad}} \widehat{\psi}_{s}\right)_{L^{2}\left(S_{x_{3}}\right)}=1$

(ii) $\left(\frac{\partial}{\partial x_{3}} \operatorname{rot} \widehat{\varphi}_{s}, \widetilde{\operatorname{grad}} \widehat{\psi}_{s}\right)_{L^{2}\left(S_{x_{3}}\right)}+\left(\int_{\partial S_{x_{3}}} g \operatorname{grad} \widehat{\varphi}_{s} \cdot \overline{\widetilde{\operatorname{rot}} \widehat{\psi}_{s}}\right)=0$

(iii) $\left(\frac{\partial}{\partial x_{3}} \widetilde{\operatorname{rot}} \widehat{\psi}_{s}, \operatorname{grad} \widehat{\varphi}_{s}\right)_{L^{2}\left(S_{x_{3}}\right)}=0$. 
Proof $(i)$ We only prove the first equality, the second one easily follows by (8). We first note that since $\Gamma$ has $(2 \mathrm{D})$ measure 0 , we can write

$$
\left(\widetilde{\operatorname{rot}} \widehat{\psi}_{s}, \operatorname{grad} \widehat{\varphi}_{s}\right)_{L^{2}\left(S_{x_{3}}\right)}=\int_{S_{x_{3}}^{\Gamma}} \operatorname{rot} \widehat{\psi}_{s} \cdot \overline{\operatorname{grad} \widehat{\varphi}_{s}} d \mathbf{x}_{T}
$$

Next we apply Green's formula $\left(10\right.$, (ii)) with $u=\widehat{\varphi}_{s}, \mathbf{v}=\operatorname{rot} \widehat{\psi}_{s}$ and $S_{x_{3}}^{\Gamma}$ instead of $S_{x_{3}}$. Of course we have to cope with the fact that, due to the presence of the cut $\Gamma$, the domain $S_{x_{3}}^{\Gamma}$ is no longer locally on one side of its boundary. Using $\operatorname{rot} \operatorname{grad} \widehat{\varphi}_{s}=0$, the reader will verify that

$$
\left(\widetilde{\operatorname{rot}} \widehat{\psi}_{s}, \operatorname{grad} \widehat{\varphi}_{s}\right)_{L^{2}\left(S_{x_{3}}\right)}=\int_{\partial S_{x_{3}}} \widehat{\psi}_{s} \overline{\operatorname{grad} \widehat{\varphi}_{s} \times \mathbf{n}_{T}} d \sigma_{x_{3}}-\int_{\Gamma} \frac{\overline{\partial \widehat{\varphi}_{s}}}{\partial \tau} d \gamma
$$

The first term is 0 because $\widehat{\varphi}_{s}$ is constant on $S_{x_{3}}^{ \pm}$, moreover $\int_{\Gamma} \frac{\overline{\partial \widehat{\varphi}_{s}}}{\partial \tau} d \gamma=1$, since $\Gamma$ joins $S_{x_{3}}^{-}$where $\widehat{\varphi}_{s}=0$ to $S_{x_{3}}^{+}$where $\widehat{\varphi}_{s}=1$.

(ii) When computing the left hand side of (46)(ii), we can this time apply Green's formula in all $S_{x_{3}}$ because $\widehat{\varphi}_{s}$, and thus its $x_{3}$-derivative, belongs to $H^{1}\left(S_{x_{3}}\right)$. We get, using rot $\operatorname{grad} \widehat{\varphi}_{s}=0$ and the second identity in (9)

$$
\begin{aligned}
& \left(\frac{\partial}{\partial x_{3}} \operatorname{rot} \widehat{\varphi}_{s}, \widetilde{\operatorname{grad}} \widehat{\psi}_{s}\right)_{L^{2}\left(S_{x_{3}}\right)} \\
& =\int_{\partial S_{x_{3}}} \frac{\partial \widehat{\varphi}_{s}}{\partial x_{3}} \widetilde{\widetilde{\operatorname{grad}} \widehat{\psi}_{s} \times \mathbf{n}_{T}} d \sigma_{x_{3}}=-\int_{\partial S_{x_{3}}} \frac{\partial \widehat{\varphi}_{s}}{\partial x_{3}} \mathbf{n}_{T} \cdot \widetilde{\widetilde{\operatorname{rot}} \widehat{\psi}_{s}} d \sigma_{x_{3}} .
\end{aligned}
$$

Considering $\widehat{\varphi}_{s}$ as a function of $\mathbf{x} \in \Omega$, then $\widehat{\varphi}_{s}$ is constant on $\partial \Omega$, which means that, on $\partial \Omega$, the $3 \mathrm{D}$ gradient is orthogonal to $\partial \Omega$ or, equivalently, the $3 \mathrm{D}$ vector product between $\left(\operatorname{grad} \widehat{\varphi}_{s}, \partial_{x_{3}} \widehat{\varphi}_{s}\right)^{t}$ and the normal vector to $\partial \Omega$, namely $\mathbf{n}=\left(\mathbf{n}_{T}, g\right)^{t}$ (see (2) with $\delta=1$ ) is identically 0 . Projecting this equation on the $\left(x_{1}, x_{2}\right)$ plane, we get

$$
\frac{\partial \widehat{\varphi}_{s}}{\partial x_{3}} \mathbf{n}_{T}=g \operatorname{grad} \widehat{\varphi}_{s}
$$

substituting this equality into (47) leads to (46)( $i i)$.

(iii) For proving the last equality, it appears useful from the technical point 
of view to work in a weak sense and to exploit $(46)(i)$. Let $\chi=\chi\left(x_{3}\right)$ be a $1 \mathrm{D}$ test function $\left(C^{\infty}\right.$ with compact support), we multiply $(46)(i)$ by $\chi^{\prime}\left(x_{3}\right)$ and then integrate over $x_{3}$. We obtain

$$
\int_{-\infty}^{+\infty} \int_{S_{x_{3}}} \widetilde{\operatorname{rot}} \widehat{\psi}_{s} \cdot \overline{\operatorname{grad} \widehat{\varphi}_{s}} \chi^{\prime}\left(x_{3}\right) d \mathbf{x}_{T} d x_{3} \equiv \int_{\Omega} \widetilde{\operatorname{rot}} \widehat{\psi}_{s} \cdot \overline{\operatorname{grad} \widehat{\varphi}_{s}} \chi^{\prime} d \mathbf{x}=0
$$

We then use the 3D Green's formula in $\Omega$ to obtain, denoting $d \Sigma$ the surface measure on $\partial \Omega$ (we use again that $\mathbf{n}=\left(\mathbf{n}_{T}, g\right)^{t}$ is normal to $\partial \Omega$ which implies that the third component of the outgoing unit normal vector to $\partial \Omega$ is $g /|\mathbf{n}|$ )

$$
\begin{aligned}
& -\int_{\Omega} \frac{\partial}{\partial x_{3}} \widetilde{\operatorname{rot}} \widehat{\psi}_{s} \cdot \overline{\operatorname{grad} \widehat{\varphi}_{s}} \chi d \mathbf{x} \\
& \quad-\int_{\Omega} \widetilde{\operatorname{rot}} \widehat{\psi}_{s} \cdot \frac{\partial}{\partial x_{3}} \overline{\operatorname{grad} \widehat{\varphi}_{s}} \chi d \mathbf{x}+\int_{\partial \Omega} \widetilde{\operatorname{rot}} \widehat{\psi}_{s} \cdot \overline{\operatorname{grad} \widehat{\varphi}_{s}} \frac{g}{|\mathbf{n}|} \chi d \Sigma=0 .
\end{aligned}
$$

Finally, as $d \sigma_{x_{3}}$ denoted the lineic measure along $\partial S_{x_{3}}$, one easily checks the formula

$$
d \Sigma=|\mathbf{n}| d \sigma_{x_{3}} d x_{3}
$$

so that the previous equality becomes

$$
\begin{aligned}
& \int_{-\infty}^{+\infty}\left(\int_{S_{x_{3}}} \frac{\partial}{\partial x_{3}} \widetilde{\operatorname{rot}} \widehat{\psi}_{s} \cdot \overline{\operatorname{grad} \widehat{\varphi}_{s}} d \mathbf{x}_{T}+\int_{S_{x_{3}}} \widetilde{\operatorname{rot}} \widehat{\psi}_{s} \cdot \frac{\partial}{\partial x_{3}} \overline{\operatorname{grad} \widehat{\varphi}_{s}} d \mathbf{x}_{T}\right. \\
& \left.-\int_{\partial S_{x_{3}}} \widetilde{\operatorname{rot}} \widehat{\psi}_{s} \cdot \overline{\operatorname{grad} \widehat{\varphi}_{s}} g d \sigma_{x_{3}}\right) \chi\left(x_{3}\right) d x_{3}=0
\end{aligned}
$$

that is to say, the above equality being valid for any test function $\chi$,

$$
\begin{aligned}
& \int_{S_{x_{3}}} \frac{\partial}{\partial x_{3}} \widetilde{\operatorname{rot}} \widehat{\psi}_{s} \cdot \overline{\operatorname{grad} \widehat{\varphi}_{s}} d \mathbf{x}_{T}+ \\
& \quad \int_{S_{x_{3}}} \widetilde{\operatorname{rot}} \widehat{\psi}_{s} \cdot \frac{\partial}{\partial x_{3}} \overline{\operatorname{grad} \widehat{\varphi}_{s}} d \mathbf{x}_{T}-\int_{\partial S_{x_{3}}} \widetilde{\operatorname{rot}} \widehat{\psi}_{s} \cdot \overline{\operatorname{grad} \widehat{\varphi}_{s}} g d \sigma_{x_{3}}=0
\end{aligned}
$$


Using (8) for the second term, this gives

$$
\begin{aligned}
& \left(\frac{\partial}{\partial x_{3}} \widetilde{\operatorname{rot}} \widehat{\psi}_{s}, \operatorname{grad} \widehat{\varphi}_{s}\right)_{L^{2}\left(S_{x_{3}}\right)} \\
& \quad=\left(\widetilde{\operatorname{grad}} \widehat{\psi}_{s}, \frac{\partial}{\partial x_{3}} \operatorname{rot} \widehat{\varphi}_{s}\right)_{L^{2}\left(S_{x_{3}}\right)}+\int_{\partial S_{x_{3}}} \widetilde{\operatorname{rot}} \widehat{\psi}_{s} \cdot \overline{\operatorname{grad} \widehat{\varphi}_{s}} g d \sigma_{x_{3}}
\end{aligned}
$$

which implies $(46)(i i i)$ by $(46)(i i)$.

Finally using the identities (46) in (45), we obtain the homogenized 1D model in the frequency domain, for all $x_{3} \in \mathbb{R}$

$$
\left\{\begin{array}{l}
\left(i \omega \widehat{C}\left(x_{3}, \omega\right)+\widehat{G}\left(x_{3}, \omega\right)\right) \widehat{V}\left(x_{3}, \omega\right)+\frac{\partial \widehat{I}}{\partial x_{3}}\left(x_{3}, \omega\right)=\widehat{j}\left(x_{3}, \omega\right), \\
\left(i \omega \widehat{L}\left(x_{3}, \omega\right)+\widehat{R}\left(x_{3}, \omega\right)\right) \widehat{I}\left(x_{3}, \omega\right)+\frac{\partial \widehat{V}}{\partial x_{3}}\left(x_{3}, \omega\right)=0
\end{array}\right.
$$

where the homogenized coefficients $\left(\widehat{C}\left(x_{3}, \omega\right), \widehat{L}\left(x_{3}, \omega\right), \widehat{G}\left(x_{3}, \omega\right), \widehat{R}\left(x_{3}, \omega\right)\right)$ are given by (37)-(40) and where the source term $\widehat{j}\left(x_{3}, \omega\right)$ is given by

$$
\widehat{j}\left(x_{3}, \omega\right)=\left(\widehat{\mathbf{j}}_{T}\left(x_{3}, \omega\right), \operatorname{grad} \widehat{\varphi}_{s}\left(x_{3}, \omega\right)\right)_{L^{2}\left(S_{x_{3}}\right)} .
$$

\section{Properties of the homogenized coefficients}

We study the behavior, for large $|\omega|$ of $\widehat{\varphi}_{s}\left(\cdot, x_{3}, \omega\right)$ and $\widehat{\psi}_{s}\left(\cdot, x_{3}, \omega\right)$. The next two lemmas are stated without their proof, which is quite straightforward.

Lemma 4.1. One has the decomposition

$$
\widehat{\varphi}_{s}\left(\cdot, x_{3}, \omega\right)=\varphi_{s}^{\infty}\left(\cdot, x_{3}\right)+\widehat{\varphi}_{r}\left(\cdot, x_{3}, \omega\right) \text { in } H^{1}\left(S_{x_{3}}\right),
$$

where the real valued function $\varphi_{s}^{\infty}\left(\cdot, x_{3}\right)$ is the unique solution of (29) and the "rest" $\widehat{\varphi}_{r}\left(\cdot, x_{3}, \omega\right)$ satisfies:

$$
\forall x_{3} \in \mathbb{R}, \quad\left\|\operatorname{grad} \widehat{\varphi}_{r}\left(\cdot, x_{3}, \omega\right)\right\|_{L^{2}\left(S_{x_{3}}\right)} \leq C\left(x_{3}\right)|\omega|^{-1},
$$

where $C\left(x_{3}\right)$ is a strictly positive function of $x_{3}$ independent of $\omega$. 
Remark that $\widehat{\varphi}_{r} \equiv \widehat{\varphi}_{r}\left(\cdot, x_{3}, \omega\right) \in H^{1}\left(S_{x_{3}}\right)$ defined by (49) satisfies

$$
\begin{cases}\operatorname{div}\left(\left(i \omega \varepsilon+\sigma_{e}\right) \operatorname{grad} \widehat{\varphi}_{r}\right)=-\operatorname{div}\left(\sigma_{e} \operatorname{grad} \varphi_{s}^{\infty}\right) & \text { in } S_{x_{3}}, \\ \widehat{\varphi}_{r}=0, & \text { on } \partial S_{x_{3}} .\end{cases}
$$

Lemma 4.2. One has the decomposition

$$
\widehat{\psi}_{s}\left(\cdot, x_{3}, \omega\right)=\psi_{s}^{\infty}\left(\cdot, x_{3}\right)+\widehat{\psi}_{r}\left(\cdot, x_{3}, \omega\right) \text { in } H^{1}\left(S_{x_{3}}^{\Gamma}\right),
$$

where the real valued function $\psi_{s}^{\infty}\left(\cdot, x_{3}\right)$ is the unique solution of (33) and the "rest" $\widehat{\psi}_{r}\left(\cdot, x_{3}, \omega\right)$ satisfies:

$$
\forall x_{3} \in \mathbb{R}, \quad\left\|\operatorname{grad} \widehat{\psi}_{r}\left(\cdot, x_{3}, \omega\right)\right\|_{L^{2}\left(S_{x_{3}}\right)} \leq C\left(x_{3}\right)|\omega|^{-1},
$$

where $C\left(x_{3}\right)$ is a strictly positive function of $x_{3}$ independent of $\omega$.

Note that $\widehat{\psi}_{r} \equiv \widehat{\psi}_{r}\left(\cdot, x_{3}, \omega\right) \in H^{1}\left(S_{x_{3}}\right)$ satisfies $\int_{S_{x_{3}}} \widehat{\psi}_{r} d \mathbf{x}_{T}=0$ and

$$
\begin{cases}\operatorname{div}\left(\left(i \omega \mu+\sigma_{m}\right) \operatorname{grad} \widehat{\psi}_{r}\right)=-\operatorname{div}\left(\sigma_{m} \widetilde{\operatorname{grad}} \psi_{s}^{\infty}\right) & \text { in } S_{x_{3}}, \\ \frac{\partial \widehat{\psi}_{r}}{\partial \mathbf{n}}=0, & \text { on } \partial S_{x_{3}} .\end{cases}
$$

We are now in position to state the main theorem of this section

Lemma 4.3. The homogenized coefficients defined by (37)-(40) satisfy

(i) $i \omega C\left(x_{3}, \omega\right)+G\left(x_{3}, \omega\right)=i \omega C^{\infty}\left(x_{3}\right)+G^{\infty}\left(x_{3}\right)+\widehat{k}_{e}\left(x_{3}, \omega\right)$,

(ii) $i \omega L\left(x_{3}, \omega\right)+R\left(x_{3}, \omega\right)=i \omega L^{\infty}\left(x_{3}\right)+R^{\infty}\left(x_{3}\right)+\widehat{k}_{m}\left(x_{3}, \omega\right)$,

where the high frequency limits $\left(C^{\infty}\left(x_{3}\right), L^{\infty}\left(x_{3}\right), G^{\infty}\left(x_{3}\right), R^{\infty}\left(x_{3}\right)\right)$ are

$$
\begin{aligned}
C^{\infty}\left(x_{3}\right) & \equiv \int_{S_{x_{3}}} \varepsilon\left(\mathbf{x}_{T}, x_{3}\right)\left|\operatorname{grad} \varphi_{s}^{\infty}\left(\mathbf{x}_{T}, x_{3}\right)\right|^{2} d \mathbf{x}_{T}, \\
L^{\infty}\left(x_{3}\right) & \equiv \int_{S_{x_{3}}} \mu\left(\mathbf{x}_{T}, x_{3}\right)\left|\widetilde{\operatorname{grad}} \psi_{s}^{\infty}\left(\mathbf{x}_{T}, x_{3}\right)\right|^{2} d \mathbf{x}_{T}, \\
G^{\infty}\left(x_{3}\right) & \equiv \int_{S_{x_{3}}} \sigma_{e}\left(\mathbf{x}_{T}, x_{3}\right)\left|\operatorname{grad} \varphi_{s}^{\infty}\left(x, x_{3}\right)\right|^{2} d \mathbf{x}_{T}, \\
R^{\infty}\left(x_{3}\right) & \equiv \int_{S_{x_{3}}} \sigma_{m}\left(\mathbf{x}_{T}, x_{3}\right)\left|\widetilde{\operatorname{grad}} \psi_{s}^{\infty}\left(\mathbf{x}_{T}, x_{3}\right)\right|^{2} d \mathbf{x}_{T},
\end{aligned}
$$


and where the functions $\widehat{k}_{e}\left(x_{3}, \omega\right)$ and $\widehat{k}_{m}\left(x_{3}, \omega\right)$ are given by

(i) $\widehat{k}_{e}\left(x_{3}, \omega\right)=\int_{S_{x_{3}}} \sigma_{e}\left(\mathbf{x}_{T}, x_{3}\right) \operatorname{grad} \varphi_{s}^{\infty}\left(\mathbf{x}_{T}, x_{3}\right) \cdot \operatorname{grad} \widehat{\varphi}_{r}\left(\mathbf{x}_{T}, x_{3}, \omega\right) d \mathbf{x}_{T}$,

(ii) $\widehat{k}_{m}\left(x_{3}, \omega\right)=\int_{S_{x_{3}}} \sigma_{m}\left(\mathbf{x}_{T}, x_{3}\right) \widetilde{\operatorname{grad}} \psi_{s}^{\infty}\left(\mathbf{x}_{T}, x_{3}\right) \cdot \operatorname{grad} \widehat{\psi}_{r}\left(\mathbf{x}_{T}, x_{3}, \omega\right) d \mathbf{x}_{T}$,

and satisfy, as functions of the frequency:

$$
\forall x_{3} \in \mathbb{R}, \quad\left(\widehat{k}_{e}\left(x_{3}, \cdot\right), \widehat{k}_{m}\left(x_{3}, \cdot\right)\right) \in L^{2}(\mathbb{R})^{2} .
$$

Proof The proof follows from a simple calculation resulting from substitution of (49) into (37)-(40). We shall omit the proofs of (53) and (55) which are relatively straighforward. To prove (56), it suffices to prove that $\omega \longrightarrow \widehat{k}_{e}\left(x_{3}, \omega\right)$ is bounded and decays fast enough for large $|\omega|$. For boundedness, we first use Cauchy-Schartz inequality to get

$$
\left|\widehat{k}_{e}\left(x_{3}, \omega\right)\right| \leq\left(\int_{S_{x_{3}}} \sigma_{e}\left|\operatorname{grad} \widehat{\varphi}_{r}\right|^{2} d \mathbf{x}_{T}\right)^{\frac{1}{2}}\left(\int_{S_{x_{3}}} \sigma_{e}\left|\operatorname{grad} \varphi_{s}^{\infty}\right|^{2} d \mathbf{x}_{T}\right)^{\frac{1}{2}}
$$

and to notice that, from the real part of the following equality

$$
\int_{S_{x_{3}}}\left(i \omega \varepsilon+\sigma_{e}\right)\left|\operatorname{grad} \widehat{\varphi}_{r}\right|^{2} d \mathbf{x}_{T}=-\int_{S_{x_{3}}} \sigma_{e} \operatorname{grad} \varphi_{s}^{\infty} \cdot \overline{\operatorname{grad} \widehat{\varphi}_{r}} d \mathbf{x}_{T} .
$$

which obtained using (50), and Cauchy-Schwartz inequality, we also have

$$
\left(\int_{S_{x_{3}}} \sigma_{e}\left|\operatorname{grad} \widehat{\varphi}_{r}\right|^{2} d \mathbf{x}_{T}\right)^{\frac{1}{2}} \leq\left(\int_{S_{x_{3}}} \sigma_{e}\left|\operatorname{grad} \varphi_{s}^{\infty}\right|^{2} d \mathbf{x}_{T}\right)^{\frac{1}{2}}
$$

Thus (57) yields : $\left|\widehat{k}_{e}\left(x_{3}, \omega\right)\right|^{2} \leq \int_{S_{x_{3}}} \sigma_{e}\left|\operatorname{grad} \varphi_{s}^{\infty}\right|^{2} d \mathbf{x}_{T}$.

Next, for large $\omega$, we deduce from (57), (58) and the range of variability of $\sigma_{e} / \varepsilon$ that $\left|\widehat{k}_{e}\left(x_{3}, \omega\right)\right| \leq C\left(x_{3}\right) /|\omega|$. This completes the proof.

Remark 4.1. It is clear on (54) that the homogenized coefficients $C^{\infty}\left(x_{3}\right)$ or $L^{\infty}\left(x_{3}\right)$ are real and positive. It is less clear, although most likely thanks 
to the non-degeneracy property (3) of $\varepsilon$ and $\mu$, that they do not degenerate, namely that they are bounded functions of $x_{3}$ that are bounded from below by a strictly positive constant. In section 5, to analyze the limit problem in a standard $L^{2}$ framework, we shall be led to make such an assumption, namely

Hypothesis 4.1. There exists $0<C_{-}^{\infty}<C_{+}^{\infty}$ and $0<L_{-}^{\infty}<L_{+}^{\infty}$ such that

$$
0<C_{-}^{\infty} \leq C^{\infty}\left(x_{3}\right) \leq C_{+}^{\infty}, \quad 0<L_{-}^{\infty} \leq L^{\infty}\left(x_{3}\right) \leq L_{+}^{\infty}, \quad \forall x_{3} \in \mathbb{R} .
$$

Our conjecture is that such assumptions are satisfied "in general", as soon as the holes $T_{z}$ do not degenerate (cf hypothesis 2.2). The question of finding explicit conditions on $\varepsilon, \mu$ and the domain $\Omega$ that would ensure hypothesis 4.1 is an interesting but delicate issue out of the scope of this paper.

Remark 4.2. One can check that if the function $\varepsilon\left(\cdot, x_{3}\right)$ and $\sigma_{e}\left(\cdot, x_{3}\right)$ (respectively $\mu\left(\cdot, x_{3}\right)$ and $\sigma_{m}\left(\cdot, x_{3}\right)$ ) are proportional (as in remark 3.1) then $\widehat{k}_{e}$ and $\widehat{k}_{m}$ vanish.

The next property whose proof is obvious (by simply taking the real parts of the equalities (53) and using the definition $(39,40)$ of $\widehat{G}\left(x_{3}, \omega\right)$ and $\widehat{R}\left(x_{3}, \omega\right)$ as well as inequalities (3)), plays an essential role in the analysis on the limit evolution problem in time domain.

Lemma 4.4. With $\sigma_{e,-}$ and $\sigma_{m,-}$ given by (3), one has for any $\left(x_{3}, \omega\right)$

$$
\begin{aligned}
& G^{\infty}\left(x_{3}\right)+\mathcal{R} e\left(\widehat{k}_{e}\left(x_{3}, \omega\right)\right) \geq \sigma_{e,-}\left\|\operatorname{grad} \widehat{\varphi}_{s}\left(\cdot, x_{3}, \omega\right)\right\|_{L^{2}\left(S_{x_{3}}\right)}^{2}, \\
& R^{\infty}\left(x_{3}\right)+\mathcal{R} e\left(\widehat{k}_{m}\left(x_{3}, \omega\right)\right) \geq \sigma_{m,-}\left\|\widetilde{\operatorname{grad}} \widehat{\psi}_{s}\left(\cdot, x_{3}, \omega\right)\right\|_{L^{2}\left(S_{x_{3}}\right)}^{2} .
\end{aligned}
$$

\section{The homogenized $1 \mathrm{D}$ problem in time-domain}

Thanks to lemma 4.3, we can rewrite the homogenized 1D problem in frequency domain (48) as

$$
\begin{cases}i \omega C^{\infty}\left(x_{3}\right) \widehat{V}+G^{\infty}\left(x_{3}\right) \widehat{V}+\widehat{k}_{e}\left(x_{3}, \omega\right) \widehat{V}+\frac{\partial \widehat{I}}{\partial x_{3}}=\widehat{j} \quad x_{3} \in \mathbb{R}, \\ i \omega L^{\infty}\left(x_{3}\right) \widehat{I}+R^{\infty}\left(x_{3}\right) \widehat{I}+\widehat{k}_{m}\left(x_{3}, \omega\right) \widehat{I}+\frac{\partial \widehat{V}}{\partial x_{3}}=0 \quad x_{3} \in \mathbb{R} .\end{cases}
$$

To come back to time domain, it is natural to introduce the inverse Fourier transforms (in time) of the functions $\widehat{k}_{e}\left(x_{3}, \omega\right)$ and $\widehat{k}_{m}\left(x_{3}, \omega\right)$. 
5.1. The kernels $k_{e}\left(x_{3}, t\right)$ and $k_{m}\left(x_{3}, t\right)$.

Let us introduce by definition the functions:

$$
\forall x_{3} \in \mathbb{R}, \quad\left(k_{e}\left(x_{3}, \cdot\right), k_{m}\left(x_{3}, \cdot\right)\right) \in L^{2}(\mathbb{R})^{2},
$$

as the inverse Fourier transforms (in time) of $\widehat{k}_{e}\left(x_{3}, \omega\right)$ and $\widehat{k}_{m}\left(x_{3}, \omega\right)$

$$
\forall x_{3} \in \mathbb{R}, \quad\left(k_{e}\left(x_{3}, \cdot\right), k_{m}\left(x_{3}, \cdot\right)\right) \equiv \mathcal{F}^{-1}\left(\widehat{k}_{e}\left(x_{3}, \cdot\right), \widehat{k}_{m}\left(x_{3}, \cdot\right)\right) .
$$

We are going to give below a characterization, directly in time domain, of the functions $k_{e}\left(x_{3}, t\right)$ and $k_{m}\left(x_{3}, t\right)$ that will demonstrate in particular that, as expected, these are causal functions. This characterization will also be useful from both theoretical and computational points of view. We first introduce, for each $x_{3}$, the bounded operator in $H_{0}^{1}\left(S_{x_{3}}\right)$ :

$$
\mathbf{A}_{e}\left(x_{3}\right) \in \mathcal{L}\left(\mathbf{V}_{e}\left(x_{3}\right)\right), \quad \mathbf{V}_{e}\left(x_{3}\right) \equiv H_{0}^{1}\left(S_{x_{3}}\right)
$$

such that, for any $\varphi_{e} \in \mathbf{V}_{e}, \Phi_{e} \equiv \mathbf{A}_{e}\left(x_{3}\right) \varphi_{e}$ if and only if

$$
\begin{cases}\operatorname{div}\left(\varepsilon \operatorname{grad} \Phi_{e}\right)=-\operatorname{div}\left(\sigma_{e} \operatorname{grad} \varphi_{e}\right) & \text { in } S_{x_{3}} \\ \Phi_{e}=0, & \text { on } \partial S_{x_{3}} .\end{cases}
$$

The reader will note that the fact that $\mathbf{A}_{e}\left(x_{3}\right)$ is well defined and bounded is a trivial consequence of the Lax-Milgram lemma, Poincaré's inequality and assumptions (3). Moreover, if $\mathbf{V}_{e}$ is equipped with the scalar product

$$
\left(\varphi_{e}, \varphi_{e}^{\prime}\right)_{\mathbf{v}_{e}\left(x_{3}\right)}:=\int_{S_{x_{3}}} \varepsilon\left(\mathbf{x}_{T}, x_{3}\right) \operatorname{grad} \varphi_{e}\left(\mathbf{x}_{T}\right) \cdot \overline{\operatorname{grad} \varphi_{e}^{\prime}\left(\mathbf{x}_{T}\right)} d \mathbf{x}_{T}
$$

then $\mathbf{A}_{e}\left(x_{3}\right)$ is positive and self-adjoint since for any $\left(\varphi_{e}, \varphi_{e}^{\prime}\right) \in \mathbf{V}_{e}\left(x_{3}\right)^{2}$

$$
\left(\mathbf{A}_{e}\left(x_{3}\right) \varphi_{e}, \varphi_{e}^{\prime}\right)_{\mathbf{V}_{e}\left(x_{3}\right)}:=\int_{S_{x_{3}}} \sigma_{e}\left(\mathbf{x}_{T}, x_{3}\right) \operatorname{grad} \varphi_{e}\left(\mathbf{x}_{T}\right) \cdot \overline{\operatorname{grad} \varphi_{e}^{\prime}\left(\mathbf{x}_{T}\right)} d \mathbf{x}_{T}
$$

In the same way, we introduce, for each $x_{3}$, the bounded operator

$$
\mathbf{A}_{m}\left(x_{3}\right) \in \mathcal{L}\left(\mathbf{V}_{m}\left(x_{3}\right)\right), \quad \mathbf{V}_{m}\left(x_{3}\right)=\left\{\varphi \in H^{1}\left(S_{x_{3}}\right) / \int_{S_{x_{3}}} \varphi d x=0\right\}
$$


such that, for any $\varphi_{m} \in \mathbf{V}_{m}\left(x_{3}\right), \Phi_{m} \equiv \mathbf{A}_{m}\left(x_{3}\right) \varphi_{m}$ if and only if

$$
\begin{cases}\operatorname{div}\left(\mu \operatorname{grad} \Phi_{m}\right)=-\operatorname{div}\left(\sigma_{m} \operatorname{grad} \varphi_{m}\right) & \text { in } S_{x_{3}}, \\ \frac{\partial \Phi_{m}}{\partial n}=0, & \text { on } \partial S_{x_{3}} .\end{cases}
$$

Note that $\mathbf{A}_{m}\left(x_{3}\right)$ is well defined and bounded as a consequence of LaxMilgram's lemma, Poincaré-Wirtinger's inequality and assumptions (4.1). Moreover, if $\mathbf{V}_{m}\left(x_{3}\right)$ is equipped with the scalar product

$$
\left(\varphi_{m}, \varphi_{m}^{\prime}\right)_{\mathbf{v}_{m}\left(x_{3}\right)}:=\int_{S_{x_{3}}} \mu\left(\mathbf{x}_{T}, x_{3}\right) \operatorname{grad} \varphi_{m}\left(\mathbf{x}_{T}\right) \cdot \overline{\operatorname{grad} \varphi_{m}^{\prime}\left(\mathbf{x}_{T}\right)} d \mathbf{x}_{T} .
$$

then $\mathbf{A}_{m}\left(x_{3}\right)$ is positive and self-adjoint since for any $\left(\varphi_{m}, \varphi_{m}^{\prime}\right) \in \mathbf{V}_{m}\left(x_{3}\right)^{2}$

$$
\left(\mathbf{A}_{m}\left(x_{3}\right) \varphi_{m}, \varphi_{m}^{\prime}\right) \mathbf{v}_{m}\left(x_{3}\right):=\int_{S_{x_{3}}} \sigma_{m}\left(\mathbf{x}_{T}, x_{3}\right) \operatorname{grad} \varphi_{m}\left(\mathbf{x}_{T}\right) \cdot \overline{\operatorname{grad} \varphi_{m}^{\prime}\left(\mathbf{x}_{T}\right)} d \mathbf{x}_{T}
$$

We also introduce $\left(\varphi_{r, 0}\left(\cdot, x_{3}\right), \psi_{r, 0}\left(\cdot, x_{3}\right)\right) \in \mathbf{V}_{e}\left(x_{3}\right) \times \mathbf{V}_{m}\left(x_{3}\right)$ as the respective solutions of

$$
\begin{aligned}
& \begin{cases}\operatorname{div}\left(\varepsilon \operatorname{grad} \varphi_{r, 0}\right)=-\operatorname{div}\left(\sigma_{e} \operatorname{grad} \varphi_{s}^{\infty}\right), & \text { in } S_{x_{3}}, \\
\varphi_{r, 0}=0, & \text { on } \partial S_{x_{3}},\end{cases} \\
& \begin{cases}\operatorname{div}\left(\mu \operatorname{grad} \psi_{r, 0}\right)=-\operatorname{div}\left(\sigma_{m} \widehat{\operatorname{grad}} \psi_{s}^{\infty}\right), & \text { in } S_{x_{3}}, \\
\frac{\partial \psi_{r, 0}}{\partial n}=0, & \text { on } \partial S_{x_{3}} .\end{cases}
\end{aligned}
$$

Finally, for each $x_{3} \in \mathbb{R}$, we introduce the functions:

$$
t \rightarrow \varphi_{r}\left(\cdot, x_{3}, t\right) \in L^{\infty}\left(\mathbb{R}, \mathbf{V}_{e}\left(x_{3}\right)\right), \quad t \rightarrow \psi_{r}\left(\cdot, x_{3}, t\right) \in L^{\infty}\left(\mathbb{R}, \mathbf{V}_{m}\left(x_{3}\right)\right)
$$

as the causal functions which are, for $t>0$ the unique solutions of the evolution problems ( $x_{3}$ playing the role of a parameter):

$$
\begin{aligned}
& \left\{\begin{array}{l}
\frac{d \varphi_{r}}{d t}\left(\cdot, x_{3}, t\right)+\mathbf{A}_{e}\left(x_{3}\right) \varphi_{r}\left(\cdot, x_{3}, t\right)=0 \text { for } t>0, \\
\varphi_{r}\left(\cdot, x_{3}, 0\right)=\varphi_{r, 0}\left(\cdot, x_{3}\right)
\end{array}\right. \\
& \left\{\begin{array}{l}
\frac{d \psi_{r}}{d t}\left(\cdot, x_{3}, t\right)+\mathbf{A}_{m}\left(x_{3}\right) \psi_{r}\left(\cdot, x_{3}, t\right)=0 \text { for } t>0 \\
\psi_{r}\left(\cdot, x_{3}, 0\right)=\psi_{r, 0}\left(\cdot, x_{3}\right)
\end{array}\right.
\end{aligned}
$$


Since the operators $\mathbf{A}_{e}\left(x_{3}\right)$ and $\mathbf{A}_{m}\left(x_{3}\right)$ are bounded, we know that, for $t>0$ :

$$
\begin{aligned}
& \varphi_{r}\left(\cdot, x_{3}, t\right)=e^{-t \mathbf{A}_{e}\left(x_{3}\right)} \varphi_{r, 0}\left(\cdot, x_{3}\right) \in C^{\infty}\left(\mathbb{R}^{+}, \mathbf{V}_{e}\left(x_{3}\right)\right), \\
& \psi_{r}\left(\cdot, x_{3}, t\right)=e^{-t \mathbf{A}_{m}\left(x_{3}\right)} \psi_{r, 0}\left(\cdot, x_{3}\right) \in C^{\infty}\left(\mathbb{R}^{+}, \mathbf{V}_{m}\left(x_{3}\right)\right),
\end{aligned}
$$

and since $\mathbf{A}_{e}\left(x_{3}\right)$ and $\mathbf{A}_{m}\left(x_{3}\right)$ are positive self-adjoint, we know that $\varphi_{r}\left(\cdot, x_{3}, t\right)$ and $\psi_{r}\left(\cdot, x_{3}, t\right)$ are bounded functions of time and more precisely that

$$
t \longrightarrow\left\|\varphi_{r}\left(\cdot, x_{3}, t\right)\right\|_{\mathbf{V}_{e}\left(x_{3}\right)} \quad \text { and } \quad t \longrightarrow\left\|\psi_{r}\left(\cdot, x_{3}, t\right)\right\|_{\mathbf{v}_{m}\left(x_{3}\right)}
$$

are decreasing functions. We state the main result of this paragraph:

Lemma 5.1. The functions $\varphi_{r}\left(\mathbf{x}_{T}, x_{3}, t\right)$ and $\psi_{r}\left(\mathbf{x}_{T}, x_{3}, t\right)$ are the inverse Fourier transform in time of the functions $\widehat{\varphi}_{r}\left(\mathbf{x}_{T}, x_{3}, \omega\right)$ and $\widehat{\psi}_{r}\left(\mathbf{x}_{T}, x_{3}, \omega\right)$ and the functions $k_{e}\left(x_{3}, t\right)$ and $k_{m}\left(x_{3}, t\right)$ are given by

$$
\left\{\begin{array}{l}
k_{e}\left(x_{3}, t\right)=\int_{S_{x_{3}}} \sigma_{e}\left(\mathbf{x}_{T}, x_{3}\right) \operatorname{grad} \varphi_{r}\left(\mathbf{x}_{T}, x_{3}, t\right) \cdot \operatorname{grad} \varphi_{s}^{\infty}\left(x, x_{3}\right) d \mathbf{x}_{T}, \\
k_{m}\left(x_{3}, t\right)=\int_{S_{x_{3}}} \sigma_{m}\left(\mathbf{x}_{T}, x_{3}\right) \operatorname{grad} \psi_{r}\left(\mathbf{x}_{T}, x_{3}, t\right) \cdot \widetilde{\operatorname{grad}} \psi_{s}^{\infty}\left(\mathbf{x}_{T}, x_{3}\right) d \mathbf{x}_{T} .
\end{array}\right.
$$

In particular, $k_{e}\left(x_{3}, t\right)$ and $k_{m}\left(x_{3}, t\right)$ are causal, $C^{\infty}$ in time and satisfy

$$
\begin{aligned}
& k_{e}\left(x_{3}, 0\right)=-\int_{S_{x_{3}}} \varepsilon\left(\mathbf{x}_{T}, x_{3}\right)\left|\operatorname{grad} \varphi_{r, 0}\left(\mathbf{x}_{T}\right)\right|^{2} d \mathbf{x}_{T} \leq 0, \\
& k_{m}\left(x_{3}, 0\right)=-\int_{S_{x_{3}}} \mu\left(\mathbf{x}_{T}, x_{3}\right)\left|\operatorname{grad} \psi_{r, 0}\left(x_{T}\right)\right|^{2} d \mathbf{x}_{T} \leq 0 .
\end{aligned}
$$

Proof We only give the proof $\varphi_{r}\left(\cdot, x_{3}, t\right)$. Taking into account the definition of the operator $\mathbf{A}_{e}\left(x_{3}\right)$, we deduce from $(61)$ that $\varphi_{r}\left(\cdot, x_{3}, t\right)$ satisfies

$$
\operatorname{div}\left[\left(\varepsilon\left(\cdot, x_{3}\right) \frac{\partial}{\partial t}+\sigma_{e}\left(\cdot, x_{3}\right)\right) \operatorname{grad} \varphi_{r}\left(\cdot, x_{3}, t\right)\right]=0, \quad \text { in } S_{x_{3}} \times \mathbb{R}^{+} .
$$

We deduce that the time Fourier transform of $\varphi_{r}\left(\cdot, x_{3}, t\right)$, denoted $\widetilde{\varphi}_{r}\left(\cdot, x_{3}, \omega\right)$ satisfies, using $\varphi_{r}\left(\cdot, x_{3}, 0\right)=\varphi_{r, 0}\left(\cdot, x_{3}\right)$ and $(60)$

$$
\operatorname{div}\left[\left(i \omega \varepsilon+\sigma_{e}\right) \operatorname{grad} \widetilde{\varphi}_{r}\right]=\operatorname{div}\left(\varepsilon \operatorname{grad} \varphi_{r, 0}\right) \equiv-\operatorname{div}\left(\sigma_{e} \operatorname{grad} \varphi_{s}^{\infty}\right)
$$


as $\widetilde{\varphi}_{r}\left(\cdot, x_{3}, \omega\right)=0$ on $\partial S_{x_{3}}$, comparing (65) with (50), we conclude by a uniqueness argument that $\widetilde{\varphi}_{r}\left(\cdot, x_{3}, \omega\right)=\widehat{\varphi}_{r}\left(\cdot, x_{3}, \omega\right)$. Then, formulas (63) are a consequence of (55) and of the linearity of the Fourier transform, the causality and regularity of $k_{e}\left(x_{3}, t\right)$ and $k_{m}\left(x_{3}, t\right)$ follow from the similar properties of $\varphi_{r}\left(\cdot, x_{3}, t\right)$ and $\psi_{r}\left(\cdot, x_{3}, t\right)$.

The (easy) proof of (64) is left to the reader.

\subsection{The 1D effective model in time domain}

Our goal is to identify the (transverse) limit fields $E_{T}^{0}$ and $H_{T}^{0}$. Denoting $V\left(x_{3}, t\right)$ and $I\left(x_{3}, t\right)$ the inverse Fourier transforms of the functions $\widehat{V}\left(x_{3}, \omega\right)$ and $\widehat{I}\left(x_{3}, \omega\right)$ (introduced in section 3.1), we deduce from (27) and (32), lemmas 4.1, 4.2 and 5.1 as well as (62) that

$$
\left\{\begin{aligned}
E_{T}^{0}\left(\mathbf{x}_{T}, x_{3}, t\right) & =V\left(x_{3}, t\right) \operatorname{grad} \varphi_{s}^{\infty}\left(\mathbf{x}_{T} / \delta, x_{3}\right) \\
& +\int_{0}^{t} V\left(x_{3}, s\right) \operatorname{grad} \varphi_{r}\left(\mathbf{x}_{T} / \delta, x_{3}, t-s\right) d s \\
H_{T}^{0}\left(\mathbf{x}_{T}, x_{3}, t\right) & =I\left(x_{3}, t\right) \widetilde{\operatorname{grad}} \psi_{s}^{\infty}\left(\mathbf{x}_{T} / \delta, x_{3}\right) \\
& +\int_{0}^{t} I\left(x_{3}, s\right) \operatorname{grad} \psi_{r}\left(\mathbf{x}_{T} / \delta, x_{3}, t-s\right) d s
\end{aligned}\right.
$$

where the functions $\varphi_{s}^{\infty}$ and $\psi_{s}^{\infty}$ are defined by $(29,33)$ and the functions $\varphi_{r}$ and $\psi_{r}$ by $(60,61)$. From the properties of the Fourier transform and lemma 5.1 , we deduce from the frequency domain equations (59) that $V\left(x_{3}, t\right)$ and $I\left(x_{3}, t\right)$ satisfiy the integro-differential equations, for $x_{3} \in \mathbb{R}, t>0$,

$$
\left\{\begin{array}{c}
C^{\infty}\left(x_{3}\right) \frac{\partial V}{\partial t}+G^{\infty}\left(x_{3}\right) V+\frac{\partial I}{\partial x_{3}}\left(x_{3}, t\right)+k_{e}\left(x_{3}, \cdot\right) * V\left(x_{3}, \cdot\right)=j \\
L^{\infty}\left(x_{3}\right) \frac{\partial I}{\partial t}+R^{\infty}\left(x_{3}\right) I+\frac{\partial V}{\partial x_{3}}\left(x_{3}, t\right)+k_{m}\left(x_{3}, \cdot\right) * I\left(x_{3}, \cdot\right)=0
\end{array}\right.
$$

with zero initial conditions (as a trivial consequence of (6))

$$
V\left(x_{3}, 0\right)=0, \quad I\left(x_{3}, 0\right)=0 .
$$

In $(67), *$ holds for time convolution, the convolution kernels $k_{e}\left(x_{3}, t\right)$ and $k_{m}\left(x_{3}, t\right)$ are characterized in lemma 5.1 and the "high frequency" homogenized coefficients $C^{\infty}\left(x_{3}\right), L^{\infty}\left(x_{3}\right)$ and so on are given by (54). Equation (67) 
is a general Telegraphist's equation due to the presence of additional convolution terms whose existence require both losses and heterogeneity of the cross section (see remark 4.2). To guarantee that the equations (66) characterize the limit (rescaled) electric and magnetic fields, it suffices to prove that the evolution problem $(67,68)$ is well-posed. This is possible under hypothesis 4.1 .

Theorem 5.1. Assume that hypothesis 4.1 holds and $j \in L_{\text {loc }}^{1}\left(\mathbb{R}^{+}, L^{2}(\mathbb{R})\right)$. Then, the evolution problem $(67,68)$ admits a unique (weak) solution

$$
(V, I) \in C^{0}\left(\mathbb{R}^{+} ; L^{2}(\mathbb{R})\right)
$$

that also satisfies the a priori estimate, for any $T>0$

$$
\forall t \leq T, \quad \int_{\mathbb{R}}\left(\left|V\left(x_{3}, T\right)\right|^{2}+\left|I\left(x_{3}, T\right)\right|^{2}\right) d x_{3} \leq C\|j\|_{L^{1}\left(0, T, L^{2}(\mathbb{R})\right)} .
$$

We shall not derive the technical details of the proof that can be achieved in different ways, for instance using a Galerkin method as in [4]. The key point is the (formal) derivation of the a priori estimate (69) that relies on a coercivity property of the convolution operators appearing in (67).

Lemma 5.2. For any $x_{3} \in \mathbb{R}$, any $T>0$ and any $\xi(t) \in L^{2}\left(\mathbb{R}^{+}\right)$, one has

$$
\begin{aligned}
& G^{\infty}\left(x_{3}\right) \int_{0}^{T}|\xi(t)|^{2} d t+\int_{0}^{T} \int_{0}^{t} k_{e}\left(x_{3}, t-s\right) \xi(s) \xi(t) d s d t \geq 0 \\
& R^{\infty}\left(x_{3}\right) \int_{0}^{T}|\xi(t)|^{2} d t+\int_{0}^{T} \int_{0}^{t} k_{m}\left(x_{3}, t-s\right) \xi(s) \xi(t) d s d t \geq 0 .
\end{aligned}
$$

Proof The proof is based on causality arguments and Plancherel's theorem. Lemma 4.4 and Plancherel's theorem. We omit the details.

With the help of Lemma 5.2, the formal derivation of the estimate (69) follows a standard energy approach. The model (67) is a lower order perturbation of a $1 \mathrm{D}$ wave equation with variable (in $x_{3}$ ) velocity given by

$$
c^{\infty}\left(x_{3}\right)=\left(C^{\infty}\left(x_{3}\right) L^{\infty}\left(x_{3}\right)\right)^{-\frac{1}{2}} .
$$

It is easy to determine an upper bound for $c^{\infty}\left(x_{3}\right)$, from the variable velocity for 3D Maxwell's equation in the domain $\Omega$, namely the bounded function:

$$
c\left(\mathbf{x}_{T}, x_{3}\right)=\left(\varepsilon\left(\mathbf{x}_{T}, x_{3}\right) \mu\left(\mathbf{x}_{T}, x_{3}\right)\right)^{-\frac{1}{2}} .
$$




\section{Theorem 5.2.}

$$
\forall x_{3} \in \mathbb{R}, \quad c^{\infty}\left(x_{3}\right) \leq c^{+}\left(x_{3}\right) \equiv \sup _{\mathbf{x}_{T} \in S_{x_{3}}} c\left(\mathbf{x}_{T}, x_{3}\right)
$$

Proof In the same way that we proved (46)(i), one easily shows that (this can also be obtained by taking the limit in (46)(i) when $\omega \longrightarrow+\infty)$ :

$$
\int_{S_{x_{3}}} \operatorname{grad} \varphi_{s}^{\infty}\left(\mathbf{x}_{T}, x_{3}\right) \cdot \widetilde{\operatorname{rot}} \psi_{s}^{\infty}\left(\mathbf{x}_{T}, x_{3}\right) d \mathbf{x}_{T}=1 .
$$

Since $\left|\operatorname{rot} \psi_{s}^{\infty}\left(\mathbf{x}_{T}, x_{3}\right)\right|=\left|\operatorname{grad} \psi_{s}^{\infty}\left(\mathbf{x}_{T}, x_{3}\right)\right|$, by writing

$$
d \mathbf{x}_{T}=c\left(\mathbf{x}_{T}, x_{3}\right) \varepsilon\left(\mathbf{x}_{T}, x_{3}\right)^{\frac{1}{2}} \mu\left(\mathbf{x}_{T}, x_{3}\right)^{\frac{1}{2}} d \mathbf{x}_{T}
$$

and using Cauchy-Schwartz inequality in (71), we find

$$
1 \leq c^{+}\left(x_{3}\right) C^{\infty}\left(x_{3}\right)^{\frac{1}{2}} L^{\infty}\left(x_{3}\right)^{\frac{1}{2}}
$$

that is to say $(70)$.

This theorem says that waves in the 1D limit model cannot propagate more rapidly that in the original $3 \mathrm{D}$ domain. It appears much less obvious to obtain a lower bound for $c^{\infty}\left(x_{3}\right)$, for instance $c^{\infty}\left(x_{3}\right) \geq c^{-}\left(x_{3}\right)$, the infimum of $c\left(\mathbf{x}_{T}, x_{3}\right)$ in the cross-section $S_{x_{3}}$, which we expect to be true.

Remark 5.1. In the case of homogeneous cross sections (Example 2.3), using remark 3.3), it is easy to show that

$$
c^{\infty}\left(x_{3}\right)=\left(\varepsilon\left(x_{3}\right) \mu\left(x_{3}\right)\right)^{-\frac{1}{2}} .
$$

\subsection{A "simple" case of application.}

We conclude this section by a simple case of application for which everything can be made much more explicit. It is the case where each cross section $S_{x_{3}}$ is made of two concentric homogeneous annuli (see Figure 2 right picture). In what follows everything may depend on $x_{3}$ but, for simplicity of notation, we shall omit to mention the $x_{3}$ dependency. More precisely, let $\alpha_{1}>1$ and $\alpha_{2}>1, S\left(\equiv S_{x_{3}}\right)$ is defined by $\bar{S}=\bar{S}_{1} \cup \bar{S}_{2}$,

$$
S_{1}=\left\{\mathbf{x}_{T} \in \mathbb{R}^{2} / 1<r<\alpha_{1}\right\}, \quad S_{2}=\left\{\mathbf{x}_{T} \in \mathbb{R}^{2} / \alpha_{1}<r<\alpha_{2} \alpha_{1}\right\},
$$


where we have used the polar coordinates $(r, \theta)$ in the $\left(x_{1}, x_{2}\right)$ plane. We assume that the physical coefficients satisfy

$$
\left(\varepsilon, \mu, \sigma_{e}, \sigma_{m}\right)(x)=\left(\varepsilon_{1}, \mu_{1}, \sigma_{e, 1}, \sigma_{m, 1}\right) \text { in } S_{1}, \quad\left(\varepsilon_{2}, \mu_{2}, \sigma_{e, 2}, \sigma_{m, 2}\right) \text { in } S_{2} .
$$

In such situation it is natural to look for solutions $\left(\varphi_{s}^{\infty}, \psi_{s}^{\infty}\right)$ of problem (29) and (33) that are independent of the variable $\theta$ which leads to solving simple ODE's in the $r$ variable. After some computations, we find

$$
\operatorname{grad} \varphi_{s}^{\infty}(r, \theta)=-\frac{\varepsilon_{j}}{\varepsilon_{m}} \frac{\mathbf{e}_{r}}{r} \quad \text { in } S_{j}, j=1,2, \operatorname{grad} \psi_{s}^{\infty}(r, \theta)=-\frac{\mathbf{e}_{\theta}}{2 \pi r} \text { in } S .
$$

where $\left(\mathbf{e}_{r}, \mathbf{e}_{\theta}\right)$ is the usual orthonormal basis associated to polar coordinates and $\varepsilon_{m}$ is given by: $\varepsilon_{m}=\varepsilon_{2} \ln \alpha_{1}+\varepsilon_{1} \ln \alpha_{2}$. Let us emphasize that $\operatorname{grad} \psi_{s}^{\infty}$ is constant in all of $S$ and independent of $\mu$ : this is specific to the circular symmetry. Using the above formulas in equation (54), we get the expression of the homogenized coefficients. The "magnetic" ones, $L^{\infty}$ and $R^{\infty}$, are obtained, up to the multiplicative constant $2 \pi^{-1}$ by a weighted average on $\mu$ and $\sigma_{m}$

$$
L^{\infty}=\frac{1}{2 \pi}\left(\mu_{1} \ln \alpha_{1}+\mu_{2} \ln \alpha_{2}\right), \quad R^{\infty}=\frac{1}{2 \pi}\left(\sigma_{m, 1} \ln \alpha_{1}+\sigma_{m, 2} \ln \alpha_{2}\right) .
$$

Concerning the "electric" coefficients $\left(C^{\infty}, G^{\infty}\right)$, this is slightly more complicated since the quantities that are averaged in a similar way are non linear functions of $\varepsilon$ and $\sigma_{e}$

$$
\begin{aligned}
& \left(C^{\infty}\right)^{-1}=\frac{1}{2 \pi}\left(\varepsilon_{1}^{-1} \ln \alpha_{1}+\varepsilon_{2}^{-1} \ln \alpha_{2}\right), \\
& G^{\infty}\left(C^{\infty}\right)^{-2}=\frac{1}{2 \pi}\left(\sigma_{e, 1} \varepsilon_{1}^{-2} \ln \alpha_{1}+\sigma_{e, 2} \varepsilon_{2}^{-2} \ln \alpha_{2}\right) .
\end{aligned}
$$

The reader will observe that the squared velocity for the homogenized model, $c^{\infty}$, appears as a weighted arithmetic average of the squared velocities of each homogeneous media

$$
\left(c^{\infty}\right)^{2}=\frac{\mu_{1} c_{1}^{2} \ln \alpha_{1}+\mu_{2} c_{2}^{2} \ln \alpha_{2}}{\mu_{1} \ln \alpha_{1}+\mu_{2} \ln \alpha_{2}}, \quad c_{1}^{2}=\left(\varepsilon_{1} \mu_{1}\right)^{-1}, \quad c_{2}^{2}=\left(\varepsilon_{2} \mu_{2}\right)^{-1} .
$$

In particular, as expected: $\min \left(c_{1}, c_{2}\right) \leq c^{\infty} \leq \max \left(c_{1}, c_{2}\right)$. Finally, one can compute explicitly the electric and magnetic kernels $k_{e}(t)$ and $k_{m}(t)$ from 
(63) after having determined analytically $\left(\varphi_{r}, \psi_{r}\right)$, the solutions of (61). This is easily done, again, by looking $\left(\varphi_{r}, \psi_{r}\right)$ as functions of $r$ and $\theta$. It can be shown that $k_{e}(t)=k_{e, 0} e^{-t / \tau_{e}}, \quad k_{m}(t)=0$, where

$k_{e, 0}=2 \pi \ln \alpha_{1} \ln \alpha_{2} \frac{\left(\sigma_{e, 2} \varepsilon_{1}-\sigma_{e, 1} \varepsilon_{2}\right)^{2}}{\varepsilon_{m}^{3}}, \quad \tau_{e}=\frac{\left(\varepsilon_{1} / \ln \alpha_{1}\right)+\left(\varepsilon_{2} / \ln \alpha_{e}\right)}{\left(\sigma_{e, 1} / \ln \alpha_{1}\right)+\left(\sigma_{e, 2} / \ln \alpha_{2}\right)}$.

Here again, the fact that the magnetic kernel $k_{m}(t)$ vanishes is specific to circular symmetry. Moreover, note that if $\sigma_{e, 2} / \varepsilon_{2}=\sigma_{e, 1} / \varepsilon_{1}, k_{e}(t)$ also vanishes: this is a particular case of the remark 3.1.

Note also that $k_{e}(t)$ decays exponentially, which corresponds to a "short" memory effect, as soon as $\sigma_{e, 1}>0$ or $\sigma_{e, 2}>0$. Moreover, the fact that $k_{e}(t)$ is reduced to one simple exponential allows us to rewrite (67) as the coupling between the usual telegraphist's equation coupled, via the introduction of an auxiliary variable $W$ (that will represent the nonlocal effects) to a simple linear ordinary differential equation (we reintroduce here the dependency of the coefficients with respect to $\left.x_{3}\right)$ : the equations $(72)$ can be written under the convenient form, $x_{3} \in \mathbb{R}, t>0$,

$$
\left\{\begin{aligned}
C^{\infty}\left(x_{3}\right) \frac{\partial V}{\partial t}\left(x_{3}, t\right) & +G^{\infty}\left(x_{3}\right) V\left(x_{3}, t\right) \\
& +\frac{\partial I}{\partial x_{3}}\left(x_{3}, t\right)+k_{e, 0}\left(x_{3}\right) W\left(x_{3}, t\right)=j\left(x_{3}, t\right) \\
L^{\infty}\left(x_{3}\right) \frac{\partial I}{\partial t}\left(x_{3}, t\right) & +R^{\infty}\left(x_{3}\right) I\left(x_{3}, t\right)+\frac{\partial V}{\partial x_{3}}\left(x_{3}, t\right)=0 \\
\tau_{e}\left(x_{3}\right) \frac{\partial W}{\partial t}\left(x_{3}, t\right) & +W\left(x_{3}, t\right)=\tau_{e}\left(x_{3}\right) V\left(x_{3}, t\right)
\end{aligned}\right.
$$

We conclude this section by presenting some numerical simulations realized with the above model. For simplicity we consider the problem with constant coefficients in $x_{3}$ (which corresponds to the pure cylindrical case), posed in a finite segment $[0, \ell]$ (with $\ell=10$ ) with periodic boundary conditions to simulate infinity. We consider a gaussian source with a so called "Ricker" pulse in time: $j\left(x_{3}, t\right)=g\left(x_{3}\right) r\left(t-t_{0}\right)$ with

$$
g\left(x_{3}\right) \equiv e^{-\gamma\left(x_{3}-5\right)^{2}}, \quad r(t) \equiv 2 \pi^{2}\left((t-1)^{2}-1\right) e^{-\pi^{2}\left((t-1)^{2}-1\right)},
$$

where we have chosen, arbitrarily $\gamma=-4 \ln \left(10^{-20}\right)$ and $t_{0}=1 / 10$. We consider the particular case where the only source of heterogeneity of the 
cross section is the presence of electric conductivity in the outer layer:

$$
\varepsilon_{1}=\varepsilon_{2}=\mu_{1}=\mu_{2}=1, \quad \sigma_{m, 2}=\sigma_{m, 2}=\sigma_{e, 1}=0, \quad \sigma_{e, 2}=0.5
$$

and

$$
\alpha_{1}=8 / 5, \quad \alpha_{2}=5 / 4
$$

The dissipation phenomena are thus governed by the two following time constants, setting $\tau_{\infty} \equiv\left(C^{\infty} / G^{\infty}\right)$,

$$
\tau_{\infty}=10 / 3=3.3333, \quad \tau_{e} \simeq 2.9495 .
$$

To evaluate in this case the influence on the nonlocal effects induced by the heterogeneity of the cross section we compare a numerical simulation without these effects, by taking $k_{e, 0} \equiv 0$ with a numerical simulation where $k_{e, 0}$ is given its actual value namely:

$$
k_{e, 0} \simeq-0.4946
$$

The computations are made up to the final time $T_{f}=40$, which permits to the electromagnetic waves, that propagate at speed 1, to travel along 4 times the distance $\ell$. On Figure 3, we represent snapshots of the solutions (more precisely the electric potential, at a given time, as a function of $\left.x_{3} \in[0, \ell]\right)$ at four instants near the final times $T_{f}$. The red curves correspond to the simulation without the non local term and the blue curves correspond to the full model. In the absence of the non local terms, one simply observes a decay of the amplitude of the wave without any distortion. With the full model, in addition to the pure dissipation, we observe a change of the shape of the pulse during its propagation: this is what could be called a "dispersive dissipation" phenomenon.

\section{Conclusion}

In this paper, we have derived 1D limit models for the propagation of electromagnetic waves in thin co-axial cables by means of asymptotic analysis of the original 3D model with respect to the small transverse dimensions of the cable. The resulting model is a generalized telegraphist's model whose coefficients are determined from the solutions of 2D scalar problems in normalized cross sections. In the case of heterogeneous cross sections involving lossy 

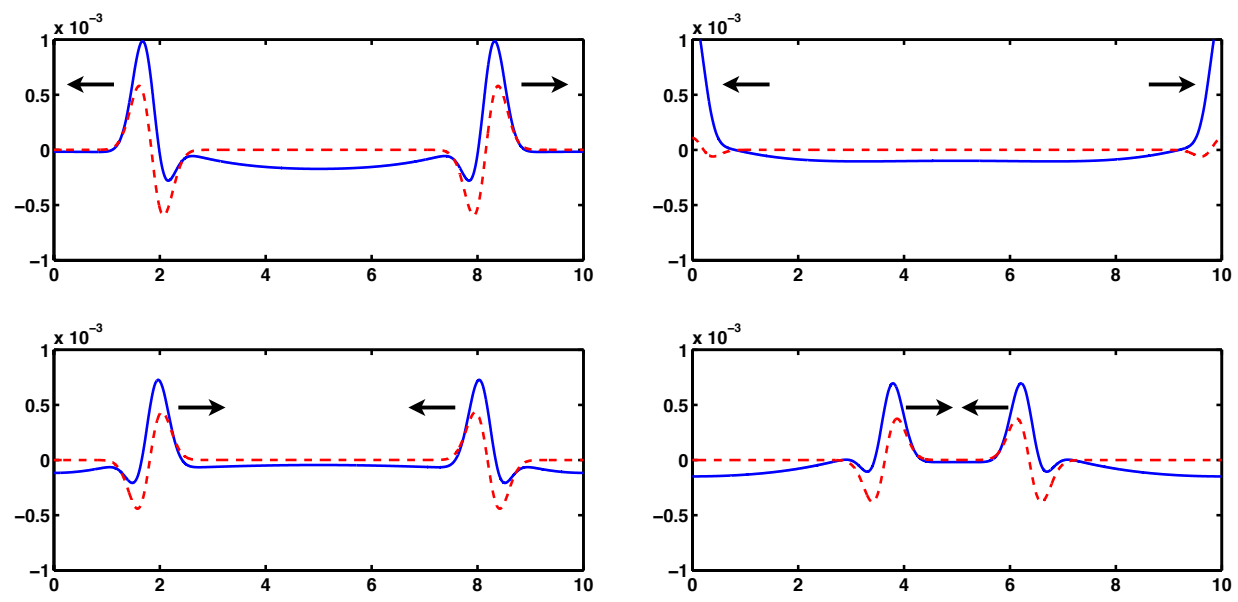

Figure 3: Snapshots of the electric potential $V\left(x_{3}, t\right)$ for $k_{e, 0} \equiv 0$ (dashed line) or $k_{e, 0} \simeq$ -0.4946 at time $t \simeq 34.33$ (upper left), $t \simeq 36.16$ (upper right), $t \simeq 37.98$ (lower left) and $t \simeq 39.81$ (lower right). To help the reader, the arrows indicate the propagation direction of the pulses.

media, additional nonlocal time convolution terms appear in the equations of the model. The next step towards the complete mathematical justification of this approximate model is the derivation of error estimates, which is the object of a forthcoming work. Among the desirable enrichments of this model let us quote:

o the derivation of more accurate (higher order) models: such models should include weak dispersive effects.

o the modeling of the junctions between several cables: the objective is to justify or generalize Kirchhoff conditions (in the spirit of [9] and [10]).

o the introduction of small defects in both the geometry and the material properties of the cable.

From a more applied point of view, another objective is the development of robust numerical methods for the solution of the limit model in the presence of convolutions term, in a more general situation than the one considered in section 5.3.

\section{References}

[1] C. Amrouche, C. Bernardi, M. Dauge, V. Girault, Vector potentials in three-dimensional non-smooth domains, M2AS 21 (1998) 823-864. 
[2] P.G. Ciarlet, Plates and Junctions in Elastic Multi-Structures: An Asymptotic Analysis (Recherches En Mathematiques Appliquees, Vol. 14), Springer-Verlag, 1991.

[3] P.G. Ciarlet, Introduction to Linear Shell Theory, Gauthier-Villars, illustrated edition edition, 1998.

[4] B. Cockburn, P. Joly, Maxwell equations in polarizable media, SIAM Journal on Mathematical Analysis 19 (1988) 1372-1390.

[5] R. Dautray, J.L. Lions, Mathematical analysis and numerical methods for science and technology. Vol. 1 and 3, Springer-Verlag, 1990.

[6] R. Dusseaux, C. Faure, Telegraphist's equations for rectangular waveguides and analysis in nonorthogonal coordinates, Progress In Electromagnetics Research 88 (2008) 53-71.

[7] S. Imperiale, P. Joly, Mathematical and numerical modelling of piezoelectric sensors, ESAIM: M2AN (2012) 875-909.

[8] S. Imperiale, P. Joly, Mathematical modeling of coaxial cables, INRIA RR, 2013.

[9] P. Joly, A. Semin, Construction and analysis of improved Kirchoff conditions for acoustic wave propagation in a junction of thin slots, ESAIM: Proc., 2008, pp. 44-67.

[10] P. Kuchment, Graph models for waves in thin structures, Waves in Random Media 12 (2002) 1-24.

[11] P. Monk, Finite element methods for Maxwell's equations, Oxford science publications, 2003.

[12] L.W. Schmerr Jr, S.J. Song, Ultrasonic Nondestructive Evaluation Systems, Springer, 2007.

[13] J. Stratton, Electromagnetic Theory, Mcgraw Hill, second printing edition, 1941.

[14] M.F. Veiga, Asymptotic method applied to a beam with a variable cross section, in: Asymptotic methods for elastic structures (Lisbon, 1993), de Gruyter, Berlin, 1995, pp. 237-254. 